TRANSACTIONS OF THE

AMERICAN MATHEMATICAL SOCIETY

Volume 349, Number 6, June 1997, Pages 2371-2389

S 0002-9947(97)01911-9

\title{
ON SINGLY-PERIODIC MINIMAL SURFACES WITH PLANAR ENDS
}

\author{
JOAQUÍN PÉREZ
}

\begin{abstract}
The spaces of nondegenerate properly embedded minimal surfaces in quotients of $\mathbf{R}^{3}$ by nontrivial translations or by screw motions with nontrivial rotational part, fixed finite topology and planar type ends, are endowed with natural structures of finite dimensional real analytic manifolds. This nondegeneracy is defined in terms of Jacobi functions. Riemann's minimal examples are characterized as the only nondegenerate surfaces with genus one in their corresponding spaces. We also give natural immersions of these spaces into certain complex Euclidean spaces which turn out to be Lagrangian immersions with respect to the standard symplectic structures.
\end{abstract}

\section{INTRODUCTION}

A large class inside the collection of properly embedded minimal surfaces in the three-dimensional Euclidean space $\mathbf{R}^{3}$ is the one consisting of those surfaces which are invariant by an infinite cyclic group of direct rigid motions $G$ acting freely on $\mathbf{R}^{3}$, called singly-periodic minimal surfaces. Such a surface can be viewed inside the flat three-manifold $\mathbf{R}^{3} / G$. In 1993, Meeks and Rosenberg [15] proved that for this kind of surfaces finiteness of the topology is equivalent to the finite total curvature assumption - the helicoid shows that this equivalence does not hold for surfaces in $\mathbf{R}^{3}$. Hence it seems natural to study these surfaces attending to their topology. At the end of the last century, Riemann [21] discovered a one-parameter family of minimal annuli $\left\{\widetilde{M}_{\lambda}\right\}_{\lambda>0}$ invariant by translations $\left\{T_{\lambda}\right\}_{\lambda>0}$, which induce properly embedded minimal tori minus two points in $\mathbf{R}^{3} /\left\langle T_{\lambda}\right\rangle$. In 1988, Karcher [11] found a one-parameter family of genus zero surfaces minus an even number of points in the quotient of $\mathbf{R}^{3}$ over the cyclic group generated by a screw motion $S_{\theta, \lambda}$ obtained by composition of a rotation of angle $\theta \in\left[0,2 \pi\left[\right.\right.$ around the $x_{3}$-axis followed by a translation of vector $(0,0, \lambda), \lambda \neq 0$. Also, Callahan, Hoffman, Karcher and Meeks $[2,3,4]$ gave, for any odd positive integer $k \geq 3$, a one-parameter family of surfaces with genus $k$ minus two points in a helicoidal quotient $\mathbf{R}^{3} / S_{\theta, \lambda}$. Uniqueness [14, 18], nonexistence [19] and structure [4] results have also been obtained for surfaces of this type. On the other hand, research has shown the importance of endowing some classes of minimal surfaces with a structure of smooth manifold [1], [20], [23], [24, 25, 26], which opens a field of possibilities by application of differential topology tools. In this line we should emphasize the work of the author and Ros

Received by the editors November 29, 1995.

1991 Mathematics Subject Classification. Primary 53A10, 53C42.

Key words and phrases. Minimal surfaces, Jacobi operator.

Research partially supported by a DGICYT Grant No. PB94-0796.

(C)1997 American Mathematical Society 
[20], where they introduce a nondegeneracy notion for a properly embedded minimal surface with finite total curvature in $\mathbf{R}^{3}$ in terms of the space of Jacobi functions on the surface which have logarithmic growth at the ends, and prove that the set of nondegenerate minimal surfaces with fixed topology is a finite dimensional realanalytic manifold. Some ideas in our paper come from the arguments in [20]; hence it could be a helpful source to the reader.

Using the description of minimal surfaces in terms of meromorphic data, Meeks and Rosenberg [15] also described the asymptotic behaviour of singly-periodic minimal surfaces, proving that they look like end of helicoids, flat vertical annuli or planes. Here we will pay attention to this last kind of surfaces.

Denote by $\mathcal{M}_{t}=\mathcal{M}_{t}(k, r)$ the space of connected orientable minimal surfaces with genus $k$ and $r$ horizontal planar ends, properly embedded in quotients of $\mathbf{R}^{3}$ over groups generated by nontrivial translations - if we avoid the trivial case of the plane, the maximum principle for minimal surfaces and Corollary 1 of [10] prove that we can suppose $k \geq 1$ and $r$ even-endowed with the uniform topology on compact sets. Given $M \in \mathcal{M}_{t}$, we describe the vector space $\mathcal{J}(M)$ of the infinitesimal deformations of $M$ in $\mathcal{M}_{t}$ and prove that $\operatorname{dim} \mathcal{J}(M) \geq 6$. If we denote by $\operatorname{period}(M)$ and flux $(M)$ the generator with positive third coordinate of the group of translations that leave $M$ invariant and the flux of the surface along a compact oriented horizontal section respectively, and by $\mathcal{M}_{t}^{*}=\left\{M \in \mathcal{M}_{t}: \operatorname{dim} \mathcal{J}(M)=\right.$ 6 and $\left.\operatorname{rank}\left(\operatorname{period}(M), \operatorname{flux}(M), e_{3}\right)>1\right\}$ the subspace of nondegenerate surfaces, then we prove that

$\mathcal{M}_{t}^{*}$ is open in $\mathcal{M}_{t}$, and in the nonvoid case, it is a six-dimensional real analytic manifold. Moreover, the tangent space at $M \in \mathcal{M}_{t}^{*}$ is given by $\mathcal{J}(M)$.

The minimal surfaces of Riemann $[9,21]$ are a one-parameter family parametrized by $\lambda>0$ in the space $\mathcal{M}_{t}^{*}$ of surfaces with genus one and two ends. More generally, we can consider any finite covering of these examples by taking a multiple of the period vector, which produces an element in the space $\mathcal{M}_{t}^{*}$ of surfaces with genus one and any even number of ends. A direct consequence of this theorem is that the corresponding one-parameter family depending on $\lambda>0$ forms a connected component in $\mathcal{M}_{t}^{*}$. In fact,

If $M \in \mathcal{M}_{t}^{*}$ is a nondegenerate surface with genus one and any even number of ends, then it is a finite covering of a Riemann example.

This assertion can be considered as an approach to the affirmative answer to the following conjecture: The classical Riemann examples and their finite coverings are the only surfaces in $\mathcal{M}_{t}$ with genus one. Related results for the two-ended case can be found in $[14,18]$. On the other hand, if $\mathcal{G}_{t}$ is the group of translations, then

$\mathcal{M}_{t}^{*} / \mathcal{G}_{t}$ is a three-dimensional real analytic manifold, and the map $f$ :

$\mathcal{M}_{t}^{*} / \mathcal{G}_{t} \longrightarrow \mathbf{R}^{6}$ given by $f([M])=(\operatorname{period}(M)$, flux $(M)),[M]$ being

the class of $M$ in $\mathcal{M}_{t}^{*} / \mathcal{G}_{t}$, is an analytic Lagrangian immersion.

A similar theory for surfaces invariant by nontrivial screw motions is developed: Let $\mathcal{M}_{s}$ be the space of connected orientable minimal surfaces with genus $k$ and $r$ horizontal planar ends, properly embedded in quotients of $\mathbf{R}^{3}$ over groups generated by nontrivial screw motions $S_{\theta, \lambda}$ with rotation angle $\left.\theta \in\right] 0,2 \pi\left[\right.$ around the $x_{3}$-axis and translation vector $(0,0, \lambda), \lambda \neq 0$, with the topology of uniform convergence on compact sets. The space $\mathcal{J}(M)$ of infinitesimal deformations now has dimension greater than or equal to four. If $S_{\theta, \lambda}, \lambda>0$, is the generator of the group of screw 
motions leaving $M$ invariant, we define $\operatorname{screw}(M)=(\theta, \lambda)$. Also, we denote by torque $_{3}(M)$, flux $_{3}(M)$ the third components of the torque and the flux vectors of $M$ along an oriented horizontal section. Now the space of nondegenerate surfaces is $\mathcal{M}_{s}^{*}=\left\{M \in \mathcal{M}_{s}: \operatorname{dim} \mathcal{J}(M)=4\right\}$, which is also open in $\mathcal{M}_{s}$. If it is nonvoid,

$\mathcal{M}_{s}^{*}$ is a four-dimensional real analytic manifold, whose tangent space at an element $M$ is $\mathcal{J}(M)$. If we denote by $\mathcal{G}_{s}$ the group generated by the rotations around the $x_{3}$-axis and the vertical translations, $\mathcal{M}_{s}^{*} / \mathcal{G}_{s}$ is a two-dimensional real analytic manifold, and the map $f: \mathcal{M}_{s}^{*} / \mathcal{G}_{s} \longrightarrow \mathbf{R}^{4}$ defined by $f([M])=\left(\operatorname{screw}(M)\right.$, torque $_{3}(M)$, flux $\left._{3}(M)\right)$ is an analytic Lagrangian immersion.

\section{Preliminary Results}

Let $\psi: \widetilde{M} \longrightarrow \mathbf{R}^{3}$ be an orientable proper minimal immersion and take a compact cycle $\Gamma \subset \widetilde{M}$. Choose a conormal unit field $\eta$ along $\Gamma$ and consider the flux of $\psi$ along $\Gamma$, that is,

$$
\operatorname{flux}(\psi, \Gamma)=\int_{\Gamma} \eta d s
$$

where $d s$ is measured in the metric induced by $\psi$. Observe that the flux does not depend on the cycle in a fixed homology class. Now take a smooth deformation $\left\{\psi_{t}\right.$ : $\left.\widetilde{M} \longrightarrow \mathbf{R}^{3}\right\}_{|t|<\varepsilon}$ by not necessarily minimal immersions —in the sense that $(t, p) \longmapsto$ $\psi_{t}(p)$ is smooth - such that $\psi_{0}=\psi$. We are interested in the velocity vector of the curve $t \longmapsto \int_{\Gamma} \eta_{t} d s_{t}$, where the subindex $\bullet_{t}$ denotes that the corresponding object is measured with respect to the metric induced by $\psi_{t}$. Although we do not require the minimality of $\psi_{t}$, we will denote the last integral by flux $\left(\psi_{t}, \Gamma\right)$. Denoting by $\gamma_{t}=\gamma_{t}\left(s_{t}\right)$ to a parametrization by the $\psi_{t}$-arc length of $\Gamma$ and by $N_{t}$ the Gauss map of $\psi_{t}$, we have

$$
\operatorname{flux}\left(\psi_{t}, \Gamma\right)=\int_{\Gamma} N_{t} \wedge D \psi_{t}\left(\gamma_{t}^{\prime}\right) d s_{t}=\int_{\Gamma} N_{t} \wedge D \psi_{t}\left(\gamma^{\prime}\right) d s
$$

We will denote the derivative at $t=0$ by a dot. Hence,

$$
\text { flix }=\int_{\Gamma} \dot{N} \wedge D \psi\left(\gamma^{\prime}\right) d s+\int_{\Gamma} N \wedge D V\left(\gamma^{\prime}\right) d s,
$$

$V$ being the variational field of $\psi_{t}$. As $\dot{N}$ is a tangent vector, the first integral can be written as

$$
\begin{aligned}
\int_{\Gamma}\left\langle\dot{N}, D \psi\left(J \gamma^{\prime}\right)\right\rangle D \psi\left(J \gamma^{\prime}\right) \wedge D \psi\left(\gamma^{\prime}\right) d s & =-\int_{\Gamma}\left\langle\dot{N}, D \psi\left(J \gamma^{\prime}\right)\right\rangle N d s \\
& =\int_{\Gamma}\left\langle N, D V\left(J \gamma^{\prime}\right)\right\rangle N d s
\end{aligned}
$$

where $J$ is the complex structure of $\widetilde{M}$. Hence we deduce that

$$
\text { flix }=\int_{\Gamma}\left[\left\langle N, D V\left(J \gamma^{\prime}\right)\right\rangle N+N \wedge D V\left(\gamma^{\prime}\right)\right] d s .
$$

In particular, flux does not depend on the variation but only on the variational field. As the last expression is linear in $V$, we can study separately the cases where $V$ is tangent or normal to $\psi$. In the first case, we can choose the variation as a 
reparametrization of $\psi$; thus its minimality implies that flux $\left(\psi_{t}, \Gamma\right)$ is constant in $t$ and flux vanishes. If $V=h N$ for a $C^{1}$-function $h$ on $\widetilde{M}$, then

$$
\begin{aligned}
\left\langle N, D V\left(J \gamma^{\prime}\right)\right\rangle N+N \wedge D V\left(\gamma^{\prime}\right) & =D h\left(J \gamma^{\prime}\right) N+h N \wedge D N\left(\gamma^{\prime}\right) \\
& =\frac{\partial h}{\partial \eta} N-h N \wedge D \psi\left(A \gamma^{\prime}\right),
\end{aligned}
$$

$A$ being the shape operator of $\psi$. As $\psi$ is minimal, it follows that $N \wedge D \psi\left(A \gamma^{\prime}\right)=$ $D \psi\left(J A \gamma^{\prime}\right)=-D \psi\left(A J \gamma^{\prime}\right)=D N\left(J \gamma^{\prime}\right)$, hence

$$
\text { flix }=\int_{\Gamma}\left(\frac{\partial h}{\partial \eta} N-h \frac{\partial N}{\partial \eta}\right) d s .
$$

This can be summarized as follows:

Lemma 1. Given a $C^{\infty}$-deformation $\left\{\psi_{t}: \widetilde{M} \longrightarrow \mathbf{R}^{3}\right\}_{|t|<\varepsilon}$ of a minimal surface $\psi_{0}=\psi$ by immersions, with variational field $V=\left.\frac{d}{d t}\right|_{t=0} \psi_{t}$, the derivative of the flux map along a compact cycle $\Gamma \subset \widetilde{M}$ is given by

$$
\left.\frac{d}{d t}\right|_{t=0} \operatorname{flux}\left(\psi_{t}, \Gamma\right)=\int_{\Gamma}\left(\frac{\partial}{\partial \eta}(\langle V, N\rangle) N-\langle V, N\rangle \frac{\partial N}{\partial \eta}\right) d s .
$$

As a consequence, if $\mathbf{a} \in \mathbf{R}^{3}$ and we take $\psi_{t}=\psi+t \mathbf{a}$ with $t$ small, the flux does not depend on $t$, so we have proved that

$$
\int_{\Gamma}\left(\frac{\partial}{\partial \eta}(\langle\mathbf{a}, N\rangle) N-\langle\mathbf{a}, N\rangle \frac{\partial N}{\partial \eta}\right) d s=0 .
$$

Let $\psi: \widetilde{M} \longrightarrow \mathbf{R}^{3}$ be a proper minimal embedding invariant by a translation of vector $T \in \mathbf{R}^{3}-\{0\}$, i.e. there exists a conformal transformation $S: \widetilde{M} \longrightarrow$ $\widetilde{M}$ such that $\psi \circ S=\psi+T$. $\psi$ induces a properly embedded minimal surface $M \subset \mathbf{R}^{3} / T$, where $M=\widetilde{M} / S$. Reciprocally, any properly embedded nonflat minimal surface $M \subset \mathbf{R}^{3} / T$ determines a connected singly-periodic minimal surface $\psi$ related with $M$ in the above way; see Meeks and Rosenberg [15]. Suppose that $M$ is orientable and has finite topology, so $M$ is homeomorphic to a finitely punctured compact surface $\bar{M}-\left\{p_{1}, \ldots, p_{r}\right\}$. It is proved in [15] that under these conditions, $M$ has finite total curvature, its conformal structure is induced by another one on $\bar{M}$, its Gauss map - which is well-defined - extends in a meromorphic way to the compactification $\bar{M}$, and $M$ has a nice behaviour at infinity: all its ends are simultaneously asymptotic to parallel planes, vertical annuli or to ends of helicoids. We are going to study the first case, which will be called, as usual, planar ends. Consider $\psi, M$ as above, such that the ends $p_{1}, \ldots, p_{r}$ of $M$ are planar. These ends lift to planar type ends in $\mathbf{R}^{3}$. Take $T$ nonhorizontal, so the ends can be supposed to be asymptotic to horizontal planes.

Lemma 2. There exist a neighbourhood $B(T)$ of $T$ in $\mathbf{R}^{3}-\{0\}$ and a $C^{\infty}$-family $\psi_{\mathbf{a}}: \widetilde{M} \longrightarrow \mathbf{R}^{3}, \mathbf{a} \in B(T)$, of singly-periodic proper immersions with ends asymptotic to horizontal planes, such that $\psi_{T}=\psi$ and $\psi_{\mathbf{a}} \circ S=\psi_{\mathbf{a}}+\mathbf{a}$, for any $\mathbf{a} \in B(T)$.

Proof. Consider the surjective linear map $\Phi: \mathcal{M}_{3}(\mathbf{R}) \longrightarrow \mathbf{R}^{3}$ given by $\Phi(B)=B T$, where $\mathcal{M}_{3}(\mathbf{R})$ denotes the set of $3 \times 3$ real matrices. Take a complement subspace $E$ of $\operatorname{Kernel}(\Phi)$ in $\mathcal{M}_{3}(\mathbf{R})$ such that $I_{3} \in E$. Hence, the $C^{\infty}$-map $\Psi: \mathbf{R}^{3} \times E \longrightarrow \mathbf{R}^{3}$ defined by $\Psi(\mathbf{a}, A)=A T-\mathbf{a}$ satisfies $\Psi\left(T, I_{3}\right)=0$ and $D_{2} \Psi_{\left(T, I_{3}\right)}$ is an isomorphism. 
The implicit function theorem gives a neighbourhood $B(T)$ of $T$ in $\mathbf{R}^{3}-\{0\}$ and a $C^{\infty}$-map $\varphi$ defined on $B(T)$ by $\mathbf{a} \in B(T) \longmapsto \varphi(\mathbf{a})=A_{\mathbf{a}} \in \mathcal{M}_{3}(\mathbf{R})$ such that $A_{\mathbf{a}} T=\mathbf{a}$ for each $\mathbf{a} \in B(T)$, and $A_{T}=I_{3}$. Take a fundamental domain $\Omega$ of $\psi$ with compact boundary contained in the union of two horizontal planes $\Pi, \Pi+T$, and consider two big finite cylinders $C_{R_{1}}, C_{R_{2}}$ parallel to the period vector $T$, of radii $R_{1}<R_{2}$, such that $\partial C_{R_{1}} \subset \Pi \cup(\Pi+T), \partial C_{R_{2}} \subset(\Pi-\varepsilon T) \cup(\Pi+(1+\varepsilon) T), \varepsilon>0$ small, and outside of $C_{R_{1}}, \psi$ is a union of horizontal graphs. Let $\Omega_{1}$ be the portion of $\Omega$ enclosed by $C_{R_{1}}$. For each $\mathbf{a} \in B(T)$ near enough to $T, A_{\mathbf{a}}\left(\Omega_{1}\right)$ is a surface with boundary contained in the solid cylinder determined by $C_{R_{2}}$, which can be extended to an a-periodic surface with boundary, contained in a certain infinite solid cylinder parallel to a. Gluing $A_{\mathbf{a}}\left(\Omega_{1}\right)$ with the ends of $\Omega$ outside $C_{R_{2}}$ we get a smooth surface between the planes $\Pi-\varepsilon T, \Pi+(1+\varepsilon) T$, that coincides with the original one outside a compact set. Extending this surface by the translation of vector $\mathbf{a}$, we find an a-periodic smooth map $\psi_{\mathbf{a}}: \widetilde{M} \longrightarrow \mathbf{R}^{3}$ with $\psi_{\mathbf{a}} \circ S=\psi_{\mathbf{a}}+\mathbf{a}$. Clearly, we can suppose that $\psi_{\mathbf{a}}$ is an immersion depending on a in a $C^{\infty}$-way and that $\psi_{T}=\psi$. Hence each $\psi_{\mathbf{a}}$ induces a properly immersed surface $M_{\mathbf{a}} \subset \mathbf{R}^{3} / \mathbf{a}$ with $r$ ends asymptotic to horizontal planes.

\section{Remark 1.}

1. If $\mathbf{a}(t),|t|<\varepsilon$, is a curve in $B(T)$ with $\mathbf{a}(0)=T$ and we denote the derivative with respect to $t$ at $t=0$ by a dot, then the linear map $\dot{\mathbf{a}} \longmapsto \dot{\psi}=\left.\frac{d}{d t}\right|_{t=0} \psi_{\mathbf{a}(t)}$ is one-to-one, because the multivaluation of $\dot{\psi}$ is given by $\dot{\psi} \circ S=\dot{\psi}+\dot{\mathbf{a}}$, as can be checked by differentiating the periodicity equation for $\psi_{\mathbf{a}}$, see lemma 2 .

2. The ends of $M_{\mathbf{a}}$ are the same as those of $M$, viewed in the different ambient spaces $\mathbf{R}^{3} / T$ and $\mathbf{R}^{3} / \mathbf{a}$. In particular all of them are minimal.

The ends of $\psi$ are planar and horizontal, i.e. they have finite total curvature, are embedded and asymptotic to horizontal planes. Hence, each one can be written as

$$
\psi(w)=\left(\frac{1}{w}, h(w)\right), \quad 0<|w|<\varepsilon,
$$

where $\varepsilon>0$ and $h$ is a smooth real valued function in $\{|w|<\varepsilon\}$, see [20]. We will call $w$ the graph coordinate at the corresponding end of $\psi$. Put $\rho=|w|$ and $d s^{2}$ the induced metric, which can be written in terms of $w$ as

$$
g_{i j}(w)=\frac{1}{\rho^{4}}\left(\delta_{i j}+\rho^{4} D_{i} h D_{j} h\right),
$$

where $\delta_{i j}=1$ if $i=j, \quad 0$ otherwise, and $D_{i} h$ denotes the corresponding first derivative of $h$. Note that the metric $d s^{2}$ can be induced on $M$ and that the graph coordinates $w, w^{\prime}$ at homologous ends of $\psi$ are related by a Möbius transformation of the type $w^{\prime}=\frac{w}{1+a w}, \quad a$ being a complex number. Take a function $\lambda \in C^{\infty}(M)$ such that around each end $p_{i}$ of $M$, it can be written as $\lambda(w)=f(w) \rho^{-4}$ in terms of the graph coordinate at any lift of the end $p_{i}$ to $\mathbf{R}^{3}$, where $f$ is a positive smooth function in $\{|w|<\varepsilon\}$, depending on the chosen lift. The above comment lets us consider such a function, and (4) implies that $d \bar{s}^{2}=\frac{1}{\lambda} d s^{2}$ is a Riemannian metric on the compactification $\bar{M}$, compatible with its complex structure. Let $N$ be the Gauss map of $\psi$, which can be induced on $M$ and extended to $\bar{M}$ by the finite total curvature assumption. Let $\widetilde{N}: \bar{M} \longrightarrow \mathbf{R}^{3}$ be a smooth map such that $\langle N, \widetilde{N}\rangle=1$ on $\bar{M}$ and $\widetilde{N}=\frac{1}{\left\langle N, e_{3}\right\rangle} e_{3}$ in a neighbourhood of each end, where $e_{3}=(0,0,1)$. 
We will also denote by $\widetilde{N}$ its $S$-invariant lift to $\widetilde{M}$. Given a near $T$, consider the a-periodic immersion $\psi_{\mathbf{a}}: \widetilde{M} \longrightarrow \mathbf{R}^{3}$ given by Lemma 2 . If $u \in C^{2, \alpha}(\bar{M})$ is small enough - here $C^{2, \alpha}(\bar{M})$ is the usual Hölder space - we can suppose that $\psi_{\mathbf{a}}+u \widetilde{N}$ is also an a-periodic immersion. Let $H\left(\psi_{\mathbf{a}}+u \tilde{N}\right)$ be its mean curvature function, which is well-defined on $M$. As the number of ends of $M$ is finite, we can suppose that the graph coordinates at the ends of a fundamental domain in the lifted surface to $\mathbf{R}^{3}$ are all defined in $0<|w|<\varepsilon$, where $\varepsilon$ is a positive real number. Now we can apply Proposition 1 in [20] to each end of this fundamental domain, and taking into account that the mean curvature function is well defined on $M$ we deduce the following assertion:

Proposition 1. There exist neighbourhoods $\mathcal{U}$ of 0 in $C^{2, \alpha}(\bar{M})$ and $\mathcal{A}$ of $T$ in $\mathbf{R}^{3}-\{0\}$ such that the map

$$
\begin{aligned}
\bar{H}: \mathcal{A} \times \mathcal{U} & \longrightarrow C^{\alpha}(\bar{M}), \\
(\mathbf{a}, u) & \longmapsto \lambda H\left(\psi_{\mathbf{a}}+u \widetilde{N}\right),
\end{aligned}
$$

is an analytic operator.

In order to recognize the differential of $\bar{H}$, take a smooth curve $t \longmapsto(\mathbf{a}(t), u(t))$ in $\mathcal{A} \times \mathcal{U}$ passing through $(T, 0)$ at $t=0$, and denote the derivative with respect to $t$ at $t=0$ by a dot. The variational field of $\psi_{\mathbf{a}(t)}+u(t) \tilde{N}$ is given by $\dot{\psi}+\dot{u} \tilde{N}$, whose normal projection is $\langle\dot{\psi}, N\rangle+\dot{u}$. Note that $\dot{u}$ is well-defined on $\bar{M}$ but this does not happen with the first term, which presents the following multivaluation:

$$
\langle\dot{\psi}, N\rangle \circ S=\langle\dot{\psi}, N\rangle+\langle\dot{\mathbf{a}}, N\rangle \text {. }
$$

Denote by $L=\Delta+\|\sigma\|^{2}=\Delta+\|\nabla N\|^{2}$ the Jacobi operator of $\psi$, where $\Delta$ is the Laplacian of $d s^{2}$ and $\|\sigma\|^{2}$ the square length of the second fundamental form of this embedding. Functions in the kernel of this operator are called Jacobi functions. It is well known that vector fields in $\mathbf{R}^{3}$ whose flow consists of dilations or isometries induce on the surface Jacobi functions, as for instance $\langle p, N\rangle$, $\operatorname{det}\left(p, N, e_{3}\right),\langle N, \mathbf{a}\rangle$, where $p$ is the position vector on $\widetilde{M}, e_{3}=(0,0,1)$ and $\mathbf{a}$ is any vector in $\mathbf{R}^{3}$. The first example is the support function of $\psi$ and the last one is usually called a linear function. We will use the same notation $L$ for the induced Jacobi operator on $M$. As $L\langle\dot{\mathbf{a}}, N\rangle=0$, the function

$$
D \bar{H}_{(T, 0)}(\dot{\mathbf{a}}, \dot{u})=\left.\lambda \frac{d}{d t}\right|_{t=0} H\left(\psi_{\mathbf{a}(t)}+u(t) \widetilde{N}\right)=\frac{1}{2} \lambda L(\langle\dot{\psi}, N\rangle+\dot{u})
$$

is well-defined on $\bar{M}$, and by Proposition 1, lies in $C^{\alpha}(\bar{M})$. On the other hand, from (5) it follows directly that the linear map $\dot{\mathbf{a}} \longmapsto\langle\dot{\psi}, N\rangle$ is injective.

Consider the vector space $V=V(M)=\left\{\langle\dot{\psi}, N\rangle=\left\langle\left.\frac{d}{d t}\right|_{t=0} \psi_{\mathbf{a}(t)}, N\right\rangle \mid \dot{\mathbf{a}} \in \mathbf{R}^{3}\right\}$ $\subset C^{\infty}(\widetilde{M})$, which can be identified with $\mathbf{R}^{3}$ by means of the above injective linear map. We can also view $C^{k, \alpha}(\bar{M})$ as $S$-invariant $C^{k, \alpha}$-functions defined on $\widetilde{M}$ which extend in a $C^{k, \alpha}$-way to the punctures. Now we define the vector space $\mathcal{B}=\mathcal{B}(M)=$ $V \oplus C^{2, \alpha}(\bar{M}) \subset C^{2, \alpha}(\widetilde{M})$, which becomes a Banach space with the natural norm on each component. (6) lets us define the following bounded linear operator:

$$
\begin{aligned}
& \bar{L}=\lambda L: \quad \mathcal{B}(M) \quad \longrightarrow \quad C^{\alpha}(\bar{M}), \\
& \langle\dot{\psi}, N\rangle+\dot{u} \longmapsto 2 D \bar{H}_{(T, 0)}(\dot{\mathbf{a}}, \dot{u}) \text {, }
\end{aligned}
$$


where $\dot{\psi}=\left.\frac{d}{d t}\right|_{t=0} \psi_{\mathbf{a}(t)}$. Hence $\bar{L}$ is the "compactification" to the Riemann surface $\bar{M}$ of the Jacobi operator $L$, i.e. $\bar{L}=\lambda L=\bar{\Delta}+\|\bar{\nabla} N\|^{2}$, where $\bar{\bullet}$ means that the corresponding object is measured respect to the metric $d \bar{s}^{2}$. This compactified Jacobi operator will play a key role in what follows, because the smoothness of the space of properly embedded minimal surfaces in quotients of $\mathbf{R}^{3}$ by translations, with fixed finite topology and planar ends, around one such surface will depend strongly on the kernel and the image of the operator $\bar{L}$ associated to the surface.

\section{The JACOBI Operator $\bar{L}$}

We continue with our properly embedded minimal surface $M \subset \mathbf{R}^{3} / T$. Take two curves $(\mathbf{a}(t), u(t)), \quad(\mathbf{b}(t), v(t))$ in a neighbourhood $\mathcal{A} \times \mathcal{U}$ of $(T, 0)$ in $\left(\mathbf{R}^{3}-\{0\}\right) \times C^{2, \alpha}(\bar{M})$ where Proposition 1 holds, such that $(\mathbf{a}(0), u(0))=$ $(\mathbf{b}(0), v(0))=(T, 0)$. In particular, $\psi_{\mathbf{a}(t)}+u(t) \widetilde{N}, \quad \psi_{\mathbf{b}(t)}+v(t) \widetilde{N}$ are proper immersions - not necessarily minimal — with ends asymptotic to horizontal planes. We will work in a fundamental domain $\Omega$ of $\psi$ whose compact boundary is contained in two horizontal planes $\Pi, \Pi+T$. Put $\partial \Omega=\Gamma \cup(\Gamma+T)$, and note that although $\dot{u}, \dot{v}$ take the same values on $\Gamma, \Gamma+T$, this is not true with $\dot{\psi}=\left.\frac{d}{d t}\right|_{t=0} \psi_{\mathbf{a}(t)}$, $\psi^{\prime}=\left.\frac{d}{d t}\right|_{t=0} \psi_{\mathbf{b}(t)}$, but their values on $\Gamma, \Gamma+T$ are related by (5). Denote by $d A, d \bar{A}$ the area elements associated to the metrics $d s^{2}, d \bar{s}^{2}$, respectively. As $\bar{L} d \bar{A}=L d A$, the divergence theorem gives

$$
\begin{aligned}
& \int_{\Omega}\left[(\langle\dot{\psi}, N\rangle+\dot{u}) \bar{L}\left(\left\langle\psi^{\prime}, N\right\rangle+\dot{v}\right)-\left(\left\langle\psi^{\prime}, N\right\rangle+\dot{v}\right) \bar{L}(\langle\dot{\psi}, N\rangle+\dot{u})\right] d \bar{A} \\
= & \int_{\partial \Omega}\left[(\langle\dot{\psi}, N\rangle+\dot{u}) \frac{\partial}{\partial \eta}\left(\left\langle\psi^{\prime}, N\right\rangle+\dot{v}\right)-\left(\left\langle\psi^{\prime}, N\right\rangle+\dot{v}\right) \frac{\partial}{\partial \eta}(\langle\dot{\psi}, N\rangle+\dot{u})\right] d s,
\end{aligned}
$$

where $\eta$ denotes the exterior conormal to $\Omega$ along its boundary. For the sake of simplicity, we will use the notation $\operatorname{flux}(\bullet)=\operatorname{flux}(\bullet, \Gamma)$ for the remainder of the paper. Using (5) and Lemma 1, the last expression can be written as

$$
\begin{gathered}
\left\langle\left.\frac{d}{d t}\right|_{t=0} \operatorname{flux}\left(\psi_{\mathbf{a}(t)}+u(t) \widetilde{N}\right), \dot{\mathbf{b}}\right\rangle-\left\langle\dot{\mathbf{a}},\left.\frac{d}{d t}\right|_{t=0} \operatorname{flux}\left(\psi_{\mathbf{b}(t)}+v(t) \widetilde{N}\right)\right\rangle \\
-\int_{\Gamma}\left(\langle\dot{\mathbf{a}}, N\rangle \frac{\partial}{\partial \eta}(\langle\dot{\mathbf{b}}, N\rangle)-\langle\dot{\mathbf{b}}, N\rangle \frac{\partial}{\partial \eta}(\langle\dot{\mathbf{a}}, N\rangle)\right) d s .
\end{gathered}
$$

Moreover, the last integral vanishes by (2), so we have obtained a skew-symmetric bilinear form defined on the space $\mathcal{B}$ which relates the differential operator $\bar{L}$ with the period and the flux maps:

Lemma 3. Let $\psi, M$ be as above. Consider a neighbourhood $\mathcal{A} \times \mathcal{U}$ of $(T, 0)$ in $\left(\mathbf{R}^{3}-\{0\}\right) \times C^{2, \alpha}(\bar{M})$ such that Proposition 1 holds. Given two curves $(\mathbf{a}(t), u(t))$, $(\mathbf{b}(t), v(t))$ in $\mathcal{A} \times \mathcal{U}$ passing through $(T, 0)$ at $t=0$, if $V_{1}, V_{2}$ denote the respective variational fields of $\psi_{\mathbf{a}(t)}+u(t) \widetilde{N}, \psi_{\mathbf{b}(t)}+v(t) \widetilde{N}$ and $\Omega$ is a fundamental domain of $\psi$, then

$$
\begin{gathered}
\int_{\Omega}\left(\left\langle V_{1}, N\right\rangle \bar{L}\left\langle V_{2}, N\right\rangle-\left\langle V_{2}, N\right\rangle \bar{L}\left\langle V_{1}, N\right\rangle\right) d \bar{A} \\
=\left\langle\left.\frac{d}{d t}\right|_{t=0} \operatorname{flux}\left(\psi_{\mathbf{a}(t)}+u(t) \widetilde{N}\right), \dot{\mathbf{b}}\right\rangle-\left\langle\dot{\mathbf{a}},\left.\frac{d}{d t}\right|_{t=0} \operatorname{flux}\left(\psi_{\mathbf{b}(t)}+v(t) \widetilde{N}\right)\right\rangle .
\end{gathered}
$$


The following step will be to determine the kernel and the image of the operator $\bar{L}$. Following the ideas in [20], we define $X^{\perp} \subset C^{\alpha}(\bar{M})$ to be the $L^{2}$-orthogonal complement in $C^{\alpha}(\bar{M})$ with respect to the metric $d \bar{s}^{2}$ of a subset $X \subset C^{\alpha}(\bar{M})$. Consider in the Banach space $\mathcal{B}$ the subspaces

$\mathcal{J}=\mathcal{J}(M)=\operatorname{Kernel}(\bar{L}), \quad \mathcal{K}=\mathcal{K}(M)=C^{2, \alpha}(\bar{M}) \cap \mathcal{J}, \quad \mathcal{K}_{0}=\mathcal{K}_{0}(M)=\bar{L}(\mathcal{B})^{\perp}$.

Hence, $\mathcal{K}$ consists of those functions in $\mathcal{J}$ which are well-defined on $\bar{M}$.

Lemma 4. In the above situation,

i) $\mathcal{K}_{0}=\left\{u \in \mathcal{K}: \int_{\Gamma}\left(\frac{\partial u}{\partial \eta} N-u \frac{\partial N}{\partial \eta}\right) d s=0\right\}$.

ii) $\operatorname{dim} \mathcal{J}=3+\operatorname{dim} \mathcal{K}_{0}$.

Remark 2. Note that the condition i) expresses that if $u \in \mathcal{K}_{0}$ is the normal part of the variational field for a deformation $\psi_{t}$ of our surface, then the derivative of the flux map along the deformation vanishes - see Lemma 1.

Proof. Standard elliptic theory $[7]$ insures that $\mathcal{K}=\operatorname{Kernel}\left(\left.\bar{L}\right|_{C^{2, \alpha}(\bar{M})}\right) \subset C^{\infty}(\bar{M})$ has finite dimension and that $\bar{L}\left(C^{2, \alpha}(\bar{M})\right)=\mathcal{K}^{\perp}$. This implies that $\mathcal{K}_{0}=\bar{L}(\mathcal{B})^{\perp} \subset$ $\bar{L}\left(C^{2, \alpha}(\bar{M})\right)^{\perp}=\mathcal{K}^{\perp \perp}=\mathcal{K}$. Now given $u \in \mathcal{K}_{0}$-in particular, $u$ is well-defined on $\bar{M}$ - we have $u \in \mathcal{K}_{0}$ if and only if

$$
\int_{\bar{M}} u \bar{L}(\langle\dot{\psi}, N\rangle+\dot{v}) d \bar{A}=0, \quad \text { for a generic }\langle\dot{\psi}, N\rangle+\dot{v} \in \mathcal{B} \text {. }
$$

By Lemma 3 with $\mathbf{a}(t) \equiv T$, this integral is equal to $\left\langle\left.\frac{d}{d t}\right|_{t=0}\right.$ flux $\left.(\psi+u(t) \tilde{N}), \dot{\mathbf{b}}\right\rangle$, where $\{u(t)\}_{t}$ is a curve in $\mathcal{U} \subset C^{2, \alpha}(\bar{M})$ passing through zero with velocity vector $u$ at $t=0$ and $\dot{\psi}=\left.\frac{d}{d t}\right|_{t=0} \psi_{\mathbf{b}(t)}$. By Lemma 1, this expression is equal to $\int_{\Gamma}\left[\langle\dot{\mathbf{b}}, N\rangle \frac{\partial u}{\partial \eta}-u \frac{\partial}{\partial \eta}(\langle\dot{\mathbf{b}}, N\rangle)\right] d s$, and $\left.i\right)$ is proved. The proof of $\left.i i\right)$ is the same as in Lemma 4, part 2, of [20], noticing that now $V$ is three-dimensional.

Remark 3. The Jacobi functions $\langle p, N\rangle$, $\operatorname{det}\left(p, N, e_{3}\right),\langle N$, a $\rangle$ lie in $\mathcal{J}$ for all $\mathbf{a} \in \mathbf{R}^{3}$, where $p$ denotes the position vector on $\widetilde{M}$. The third function is well-defined on $M$, and from Lemma 4 and (2) it follows that $\langle N, \mathbf{a}\rangle \in \mathcal{K}_{0}$, hence $\operatorname{dim} \mathcal{K}_{0} \geq 3$. However, $\operatorname{det}\left(p, N, e_{3}\right)$ is not well-defined on $M$ unless $T$ is vertical. Note that the support function $\langle p, N\rangle$ is never well-defined on $M$.

A properly embedded minimal surface $M \subset \mathbf{R}^{3} / T$ with finite topology and horizontal planar ends will be called nondegenerate if $\mathcal{K}_{0}(M)=\left\{\langle N, \mathbf{a}\rangle \mid \mathbf{a} \in \mathbf{R}^{3}\right\}$, $N$ being its Gauss map, i.e. if $\operatorname{dim} \mathcal{K}_{0}(M)=3$.

\section{The Space of minimal surfaces}

Let $\mathcal{M}$ be the space of connected nonflat properly embedded minimal surfaces $M$ in a quotient of $\mathbf{R}^{3}$ by a nonhorizontal translation $T$ - which depends on the surface - with fixed finite topology and horizontal planar ends. In order to endow $\mathcal{M}$ with a topological structure, we will discuss the relationship between the natural notions of convergence in this space. We will denote by $\widetilde{M}$ the periodic lift of an element $M \in \mathcal{M}$ to $\mathbf{R}^{3}$. Take $M_{n} \in \mathcal{M}, n=1,2, \ldots$, and $M_{\infty} \in \mathcal{M}$. 
1. We say that $\left\{M_{n}\right\}_{n}$ converges to $M_{\infty}$ smoothly if for any relatively compact subdomain $\Omega$ of $\widetilde{M}_{\infty}$, for any embedded tubular neighbourhood of $\Omega$ in $\mathbf{R}^{3}$, $\Omega(\varepsilon)=\left\{p+t N_{\infty}(p)|p \in \Omega| t \mid,<\varepsilon\right\}, \varepsilon>0$ small enough, $N_{\infty}$ being the Gauss map of $\widetilde{M}_{\infty}$, and for each $n$ large enough we have that $\Omega_{n}=\widetilde{M}_{n} \cap \Omega(\varepsilon)$ consists of a single graph over $\Omega, p \in \Omega \longmapsto p+u_{n}(p) N_{\infty}(p)$, and the sequence $\left\{u_{n}\right\}_{n}$ converges to zero in $C^{k}(\Omega)$, for any $k \geq 0$.

2. Let $\bar{M}_{n}, \bar{M}_{\infty}$ be the compactifications of $M_{n}=\widetilde{M}_{n} / T_{n}$ and $M_{\infty}=\widetilde{M}_{\infty} / T_{\infty}$. Consider the family $\psi_{\mathbf{a}}$ of Lemma 2 around $M_{\infty}$. We say that the sequence $\left\{\bar{M}_{n}\right\}_{n}$ converges to $\bar{M}_{\infty}$ smoothly if $T_{n} \rightarrow T_{\infty}$ and there exists a vector field $\widetilde{N} \in C^{\infty}\left(\bar{M}_{\infty}, \mathbf{R}^{3}\right)$ satisfying $\left\langle N_{\infty}, \widetilde{N}\right\rangle=1$ on $\bar{M}_{\infty}$ and $\widetilde{N}=\frac{1}{\left\langle N_{\infty}, e_{3}\right\rangle} e_{3}$ in a neighbourhood of each end of $M_{\infty}$, and there exists a sequence $\left\{u_{n}\right\}_{n} \subset$ $C^{\infty}\left(\bar{M}_{\infty}\right)$ such that $\widetilde{M}_{n}$ can be expressed as $\psi_{T_{n}}+u_{n} \widetilde{N}$ and $u_{n} \rightarrow 0$ in $C^{k}\left(\bar{M}_{\infty}\right)$, for any $k \geq 0$.

Theorem 1. Given $\left\{M_{n}\right\}_{n} \subset \mathcal{M}$ and $M_{\infty} \in \mathcal{M}$ with period vectors $T_{n} \rightarrow T_{\infty}$, the following assertions are equivalent:

1. $M_{n} \rightarrow M_{\infty}$ smoothly.

2. $\bar{M}_{n} \rightarrow \bar{M}_{\infty}$ smoothly.

Proof. It it obvious that $2 \Longrightarrow 1$, so suppose that we have 1 . Denote by $S_{\infty}$ a slab in $\mathbf{R}^{3}$ between two horizontal planes $\Pi, \Pi+T_{\infty}$ that determines a fundamental domain $\Omega$ of $\widetilde{M}_{\infty}$ with compact boundary. Take an infinite vertical solid cylinder $C_{R}$ of large radius $R$ containing $\partial \Omega$ in its interior, and denote $\Omega(R)=\widetilde{M}_{\infty} \cap S_{\infty} \cap C_{R}$. We suppose that for $\delta>0$ small, $C\left(M_{\infty}\right)-C(\Omega(R))<\frac{\delta}{2}$, where $C(\bullet)$ means the absolute total curvature enclosed in the corresponding domain. Let $S_{n}$ be a slab between parallel planes determining a fundamental domain on $\widetilde{M}_{n}$. We can also suppose $S_{n} \rightarrow S_{\infty}$ and that $\widetilde{M}_{n} \cap \partial S_{n}$ is compact and contained in $C_{R}$ for any $n$ large enough. Setting $\Omega_{n}(R)=\widetilde{M}_{n} \cap S_{n} \cap C_{R}$ and using 1, we can suppose that $C\left(M_{n}\right)-C\left(\Omega_{n}(R)\right)<\delta$, and that on $\partial \Omega_{n}(R) \cap \partial C_{R}$ the relation $\left|\left\langle N_{n}, e_{3}\right\rangle\right| \geq 1-\delta$ holds, $N_{n}$ being the Gauss map of $\widetilde{M}_{n}$. As $N_{n}$ is an open map and almost all the total absolute curvature of $M_{n}$ is inside $\Omega_{n}(R)$, we deduce that $\left|\left\langle N_{n}, e_{3}\right\rangle\right| \geq 1-\delta$ on $\left(\widetilde{M}_{n} \cap S_{n}\right)-\Omega_{n}(R)$; hence the projection of $\left(\widetilde{M}_{n} \cap S_{n}\right)-\Omega_{n}(R)$ on the $\left(x_{1}+i x_{2}\right)$ plane is a a proper local diffeomorphism, injective on each component. This proves that $\widetilde{M}_{n} \cap S_{n}$ is an $\widetilde{N}$-graph over $\psi_{T_{n}}\left(\widetilde{M}_{\infty}\right)$ by means of a certain function which is smooth at the punctures, for a suitable choice of a transversal map $\widetilde{N}$ with the conditions of Definition 2, where $\psi_{T_{n}}$ is the $T_{n}$-periodic immersion of Lemma 2 around $M_{\infty}$. Now the construction of $\psi_{T_{n}}$ in the proof of Lemma 2 proves that $\widetilde{M}_{n}$ is a global $\widetilde{N}$-graph over $\psi_{T_{n}}\left(\widetilde{M}_{\infty}\right)$ of a function $u_{n} \in C^{\infty}\left(\bar{M}_{\infty}\right)$. It remains to prove that $u_{n} \rightarrow 0$ in $C^{k}\left(\bar{M}_{\infty}\right)$. Our hypothesis implies that we only have to study the situation around an end $p_{i}$ of $M_{\infty}$, so consider the $w$-coordinate at a lift of this end to $\mathbf{R}^{3}$, which can be written as $w \longmapsto\left(\frac{1}{w}, h(w)\right), 0<|w|<\varepsilon, h$ being a smooth function in $\{|w|<\varepsilon\}$. We can suppose that this expression also parametrizes the corresponding end of $\psi_{T_{n}}$; see the proof of Lemma 2. Thus $\widetilde{M}_{n}$ is given by $w \longmapsto\left(\frac{1}{w}, h(w)+\frac{u_{n}(w)}{\left\langle N_{\infty}, e_{3}\right\rangle}\right), 0<|w|<\varepsilon$, and by hypothesis $\left.u_{n}\right|_{\{|w|=\varepsilon\}} \rightarrow 0$ in $C^{k}(\{|w|=\varepsilon\})$ for any $k \geq 0$. From Lemma 2 of [20] we have that the map

$E: \mathbf{R} \times C^{k, \alpha}(\{|w| \leq \varepsilon\}) \quad \longrightarrow \mathbf{R} \times C^{k-2, \alpha}(\{|w| \leq \varepsilon\}) \times C^{k, \alpha}(\{|w|=\varepsilon\})$, $(b, f) \quad \longmapsto\left(b, \frac{1}{\rho^{4}} H(b, f),\left.f\right|_{\{|w|=\varepsilon\}}\right)$, 
where $H(b, f)$ denotes the mean curvature of the immersion

$$
w \mapsto\left(\frac{1}{w},-b \log \rho+h(w)+\frac{f(w)}{\left\langle N_{\infty}, e_{3}\right\rangle}\right),
$$

$0<|w| \leq \varepsilon$, is an analytic local diffeomorphism around $(0,0)$, for any $k \geq 2$. Hence, $E\left(0, u_{n}\right)=\left(0,0,\left.u_{n}\right|_{\{|w|=\varepsilon\}}\right)$ lies in a neighbourhood of $(0,0,0)$ in $\mathbf{R} \times$ $C^{k-2, \alpha}(\{|w| \leq \varepsilon\}) \times C^{k, \alpha}(\{|w|=\varepsilon\})$ where $E$ is bijective. From the maximum principle at infinity [13] it follows that $b=0,\left.u_{n}\right|_{\{|w|=\varepsilon\}}$ uniquely determine $u_{n}(w)$, $|w| \leq \varepsilon$, so the bijective property of $E$ insures that $\left\{u_{n}\right\}_{n}$ converges to zero in $C^{k, \alpha}(\{|w| \leq \varepsilon\})$ for any $k \geq 2$, and the proof is complete.

Remark 4. In particular, Theorem 1 proves that the second sense of convergence defined above depends neither on the family $\psi_{\mathbf{a}}$ of Lemma 2 around $M_{\infty}$ nor on the transverse smooth map $\widetilde{N}$. Also, it implies that given $M \in \mathcal{M}, M \subset \mathbf{R}^{3} / T$, there exists a neighbourhood of $M$ in $\mathcal{M}$, endowed with the topology of uniform convergence on compact sets, such that each minimal surface in this neighbourhood is represented by $\psi_{\mathbf{a}}+u \widetilde{N}$ for suitable $\mathbf{a}$ in a neighbourhood $\mathcal{A}$ of $T$ in $\mathbf{R}^{3}-\{0\}$ and $u$ in a neighbourhood $\mathcal{U}$ of zero in $C^{\infty}(\bar{M})$, where $\psi$ is a lift of $M$ to $\mathbf{R}^{3}$ and $\widetilde{N}$ is a global transverse map defined as in section 1 , and in this neighbourhood, which will be denoted by $\mathcal{M} \cap(\mathcal{A} \times \mathcal{U})$, both topologies coincide.

Denote by $\mathcal{M}^{*} \subset \mathcal{M}$ the subset of nondegenerate surfaces with nonvertical period vector. Now we can prove the main result of this section:

Theorem 2. $\mathcal{M}^{*}$ is an open subset of $\mathcal{M}$. Moreover, $\mathcal{M}^{*}$ is either empty or a real analytic manifold with dimension 6.

Proof. We will adapt the ideas in the proof of Theorem 2 in [20]. Take $M \in \mathcal{M}^{*}$, $M \subset \mathbf{R}^{3} / T$. Consider neighbourhoods $\mathcal{U}$ of zero in $C^{2, \alpha}(\bar{M})$ and $\mathcal{A}$ of $T$ in $\mathbf{R}^{3}-\{0\}$ such that Proposition 1 holds, and define the map

$$
\begin{aligned}
F: \quad \mathbf{R}^{3} \times \mathcal{A} \times \mathcal{U} & \longrightarrow C^{\alpha}(\bar{M}), \\
\left(d_{1}, d_{2}, d_{3}, \mathbf{a}, u\right) & \longmapsto \bar{H}(\mathbf{a}, u)-\sum_{i=1}^{3} d_{i}\left\langle N_{\mathbf{a}, u}, e_{i}\right\rangle,
\end{aligned}
$$

where $N_{\mathbf{a}, u}$ is the Gauss map of $\psi_{\mathbf{a}}+u \widetilde{N}$ and $\left\{e_{1}, e_{2}, e_{3}\right\}$ is the usual basis in $\mathbf{R}^{3}$. Proposition 1 implies that $F$ is analytic. As $M$ is represented in $\mathcal{M} \cap(\mathcal{A} \times \mathcal{U})$ by $(T, 0)$, we have $F(0, T, 0)=0$. If $(\mathbf{a}, u) \in \mathcal{M} \cap(\mathcal{A} \times \mathcal{U})$ represents a minimal surface $M_{\mathbf{a}, u}$ near $M$ with Gauss map $N_{\mathbf{a}, u}$, the Banach space $\mathcal{B}\left(M_{\mathbf{a}, u}\right)=V\left(M_{\mathbf{a}, u}\right) \oplus$ $C^{2, \alpha}\left(\bar{M}_{\mathbf{a}, u}\right)$, where

$$
V\left(M_{\mathbf{a}, u}\right)=\left\{\left\langle\left.\frac{d}{d t}\right|_{t=0} \psi_{\mathbf{a}(t)}, N_{\mathbf{a}, u}\right\rangle \mid \dot{\mathbf{a}} \in \mathbf{R}^{3}, \mathbf{a}(0)=\mathbf{a}\right\},
$$

can be identified with $\mathbf{R}^{3} \oplus C^{2, \alpha}(\bar{M})$ by means of the isomorphisms $\dot{\mathbf{a}} \in \mathbf{R}^{3} \mapsto$ $\left\langle\left.\frac{d}{d t}\right|_{t=0} \psi_{\mathbf{a}(t)}, N_{\mathbf{a}, u}\right\rangle \in V\left(M_{\mathbf{a}, u}\right), \quad \dot{u} \in C^{2, \alpha}(\bar{M}) \mapsto \dot{u}\left\langle\widetilde{N}, N_{\mathbf{a}, u}\right\rangle$; note that $\left\langle\widetilde{N}, N_{\mathbf{a}, u}\right\rangle$ can be supposed smooth and positive everywhere. With this identification we can compute the differential of $F$ at $(0, \mathbf{a}, u)$ as

$$
D F_{(0, \mathbf{a}, u)}\left(d_{1}, d_{2}, d_{3}, v\right)=\frac{1}{2} \bar{L}_{\mathbf{a}, u} v-\sum_{i=1}^{3} d_{i}\left\langle N_{\mathbf{a}, u}, e_{i}\right\rangle,
$$


for any $\left(d_{1}, d_{2}, d_{3}\right) \in \mathbf{R}^{3}, v \in \mathcal{B}\left(M_{\mathbf{a}, u}\right)$, where $\bar{L}_{\mathbf{a}, u}$ denotes the compactified Jacobi operator of the minimal surface $M_{\mathbf{a}, u}$. We will use similar notations for the spaces $\mathcal{J}\left(M_{\mathbf{a}, u}\right), \mathcal{K}\left(M_{\mathbf{a}, u}\right)$ and $\mathcal{K}_{0}\left(M_{\mathbf{a}, u}\right)$, see section 2 . Note that $\bar{L}_{\mathbf{a}, u}\left(\mathcal{B}\left(M_{\mathbf{a}, u}\right)\right)$ contains the orthogonal complement of the finite dimensional space $\mathcal{K}\left(M_{\mathbf{a}, u}\right)$; hence

$$
\bar{L}_{\mathbf{a}, u}\left(\mathcal{B}\left(M_{\mathbf{a}, u}\right)\right)=\bar{L}_{\mathbf{a}, u}\left(\mathcal{B}\left(M_{\mathbf{a}, u}\right)\right)^{\perp \perp}=\mathcal{K}_{0}\left(M_{\mathbf{a}, u}\right)^{\perp},
$$

so we deduce that $\operatorname{Kernel}\left(D F_{(0, \mathbf{a}, u)}\right)=\{0\} \times \mathcal{J}\left(M_{\mathbf{a}, u}\right)=\mathcal{J}\left(M_{\mathbf{a}, u}\right)$. Moreover, the condition $M \in \mathcal{M}^{*}$ implies that $D F_{(0, T, 0)}$ is surjective, and from Lemma 4 its kernel has dimension 6 . The implicit function theorem insures that we can find a neighbourhood $\mathcal{W}$ of $(0, T, 0)$ in $\mathbf{R}^{3} \times \mathcal{A} \times \mathcal{U}$ such that $\mathcal{V}:=F^{-1}(0) \cap \mathcal{W}$ is a real analytic six-dimensional submanifold of $\mathcal{W}$. We claim that $\mathcal{V}$ contains only minimal surfaces. For, take an element $(\mathbf{a}, u) \in \mathcal{A} \times \mathcal{U}$ and consider the immersion $\psi_{\mathbf{a}}+u \widetilde{N}$. Take a fundamental domain $\Omega$ obtained by cutting this surface with a slab between two horizontal planes $\Pi, \Pi+\mathbf{a}$, with compact boundary $\partial \Omega=\Gamma \cup(\Gamma+\mathbf{a})$. Denote by $\Omega(R)$ the part of this fundamental domain inside an infinite vertical solid cylinder $C_{R}$ of large radius $R$, containing $\partial \Omega$ in its interior. Then

$$
\begin{aligned}
& 2 \int_{\Omega(R)} H\left(\psi_{\mathbf{a}}+u \widetilde{N}\right) N_{\mathbf{a}, u} d A_{\mathbf{a}, u}=\int_{\Omega(R)} \Delta_{\mathbf{a}, u}\left(\psi_{\mathbf{a}}+u \tilde{N}\right) d A_{\mathbf{a}, u} \\
& \quad=\int_{\partial \Omega(R) \cap \partial C_{R}} \eta_{\mathbf{a}, u} d s_{\mathbf{a}, u}+\int_{\Gamma} \eta_{\mathbf{a}, u} d s_{\mathbf{a}, u}+\int_{\Gamma+\mathbf{a}} \eta_{\mathbf{a}, u} d s_{\mathbf{a}, u},
\end{aligned}
$$

where the subindex $\bullet_{\mathbf{a}, u}$ means that the corresponding object is computed with respect to the induced metric by $\psi_{\mathbf{a}}+u \widetilde{N}$ and $\eta_{\mathbf{a}, u}$ is the exterior conormal field to $\Omega(R)$ along its boundary. The last two integrals are opposite, and if we argue as in the proof of Lemma 7 of [20] we find that the first integral converges to zero as $R$ goes to infinity - in Lemma 7 of [20] it is proved that this assertion holds for the horizontal components of this integral in a more general case, for embedded ends with finite total curvature and possibly nonzero logarithmic growth, and a careful reading of that proof insures that we can extend the property to the vertical component when we have only planar type ends. Thus the mean curvature function $\bar{H}(\mathbf{a}, u)$ is orthogonal to $\left\langle N_{\mathbf{a}, u}, e_{i}\right\rangle, i=1,2,3$, which proves our claim, so $\mathcal{V} \subset\{0\} \times \mathcal{A} \times \mathcal{U}=\mathcal{A} \times \mathcal{U}$. Moreover, as the tangent space of $\mathcal{V}$ at $M_{\mathbf{a}, u}$ is $\mathcal{J}\left(M_{\mathbf{a}, u}\right)$, we deduce that $\mathcal{V} \subset \mathcal{M}^{*}$. Hence $\mathcal{M}^{*}$ is open. This gives an analytic structure in a neighbourhood of each point of $\mathcal{M}^{*}$. We will finish the proof by showing the compatibility of all these structures. Given $\varepsilon>0$, denote by $\mathcal{M}_{\varepsilon}^{*}$ the set of surfaces $M \in \mathcal{M}^{*}$ such that the construction of the analytic neighbourhood $\mathcal{V}=\mathcal{V}_{M}$ can be done by using the graph coordinate around each end of a fundamental domain of the lifted surface to $\mathbf{R}^{3}$ - see the comment just before Proposition 1- over the punctured disk $\{0<|w|<\varepsilon\} . \mathcal{M}_{\varepsilon}^{*}$ is an open subset of $\mathcal{M}^{*}$. Surfaces in $\mathcal{M}_{\varepsilon}^{*}$ are, in particular, minimal immersions from $M=\bar{M}-\left\{p_{1}, \ldots, p_{r}\right\}$ into certain quotients of $\mathbf{R}^{3}$ by nonhorizontal translations. Given $M \in \mathcal{M}_{\varepsilon}^{*}$, take a fundamental domain of the lifted surface $\widetilde{M} \subset \mathbf{R}^{3}$. The end of this fundamental domain around $p_{1}$ can be expressed as $w \longmapsto\left(\frac{1}{w}, h(w)\right), 0<|w|<\varepsilon$, for certain $h \in C^{\infty}(\{|w|<\varepsilon\})$, and we can suppose that any minimal surface $M_{\mathbf{a}, u}$ in $\mathcal{V}_{M}$ can be written around $p_{1}$ as $w \longmapsto\left(\frac{1}{w}, h(w)+\frac{u(w)}{\left\langle N, e_{3}\right\rangle}\right), 0<|w|<\varepsilon$; see the proof of Lemma 2. Hence we can consider the analytic map

$$
\begin{aligned}
j: \mathcal{V}_{M} \subset \mathcal{M}_{\varepsilon}^{*} & \longrightarrow \mathbf{R}^{3} \times C^{2, \alpha}(\{|w|<\varepsilon\}), \\
M_{\mathbf{a}, u} & \longmapsto(\mathbf{a}, u) .
\end{aligned}
$$


We claim that $j$ is an immersion: if $v \in \mathcal{J}\left(M_{\mathbf{a}, u}\right)$ lies in the kernel of $D j_{M_{\mathbf{a}, u}}$, then $v$ is a well-defined function on $\bar{M}$ that satisfies $L v=0$ on $M$ and $v=0$ on a neighbourhood of $p_{1}$; hence $v=0$. As $\mathcal{M}^{*}$ is finite dimensional this is enough to insure that our claim holds. Now the fact that an immersion uniquely determines the structure of the immersed manifold implies that two analytic structures around $M \in \mathcal{M}^{*}$ are compatible. This completes the proof of the theorem.

Remark 5. 1. The above proof also insures that the procedure for constructing the analytic neighbourhood $\mathcal{V}_{M}$ of a surface $M \in \mathcal{M}^{*}$ does not depend on the family $\psi_{\mathbf{a}}$ of Lemma 2 nor on the transverse map $\widetilde{N}$.

2. When the period vector $T$ is vertical, the Jacobi function $\operatorname{det}\left(p, N, e_{3}\right), p$ being the position vector on the lifted surface $\widetilde{M} \subset \mathbf{R}^{3}$, is well-defined on $\bar{M}$; thus it belongs to $\mathcal{K}$. Moreover, it lies in $\mathcal{K}_{0}$ if and only if the flux of $\widetilde{M}$ along a horizontal section is vertical - this last condition is impossible, for instance, when $\bar{M}$ has genus one [19], or when the number of ends of $M$ with minimum branching order of the Gauss map is one [18]. Theorem 2 extends without changes around a nondegenerate surface with vertical period vector but nonvertical flux along a horizontal section. However, around a surface $M$ where both the period vector and the flux are vertical, the space of minimal surfaces where $\mathcal{K}_{0}(M)$ coincides with the space of functions spanned by the ones coming from Killing fields cannot be a manifold, because the dimension of that space at $M$ is strictly greater than the corresponding dimension for nearby surfaces. Examples of such surfaces can be found in [3] for any odd genus greater than or equal to three. These surfaces have two planar ends and a horizontal plane of symmetry, which forces both the period and the flux vectors to be vertical.

Riemann's examples $M_{\lambda}, \lambda>0$, see for example [9, 21], are a one-parameter family of tori with two planar ends. The extended Gauss map of such a surface $M_{\lambda}$ has its branching values on an equator of the sphere. Montiel and Ros [16] prove that in this situation the space $\mathcal{K}$ has dimension three; hence the same holds for $\mathcal{K}_{0}$ and the above theorem works. This implies that, up to translations, dilations and rotations around the $x_{3}$-axis, the only possible deformation of a Riemann example is by nearby Riemann examples; that is, they form an isolated family inside the corresponding space $\mathcal{M}$ of genus one surfaces with two ends. This result extends directly to any finite covering of Riemann examples obtained by considering a multiple of the period vector, in the space of genus one surfaces with any even number of ends. This nondegeneracy property characterizes those classical examples:

Theorem 3. If $M \in \mathcal{M}^{*}$ has genus one, then it is a finite covering of a Riemann example.

Proof. Take $M \in \mathcal{M}^{*}$ with genus one. We can parametrize our problem as follows: Let $\mathcal{L} \subset \mathbf{C}$ be the lattice generated by $\{a, 2 \pi i\}, a \in \mathbf{C}-\{0\}$, such that $\mathbf{C} / \mathcal{L}$ is conformally equivalent to the compactification $\bar{M}$ of $M$, the third coordinate of the lifted surface $\widetilde{M} \subset \mathbf{R}^{3}$ is $x_{3}(\xi)=k \operatorname{Re}(\xi), k$ being a nonzero real number, and the segment $\Gamma=\{i t \mid t \in[0,2 \pi]\}$ is applied on a closed curve on $\widetilde{M}$ which is nonnullhomotopic in the cylinder obtained by attaching the ends to $\widetilde{M}$. Let $g$ be the Gauss map stereographically projected from the north pole. In the $\xi$-coordinate, 
the Jacobi equation is

$$
u_{\xi \bar{\xi}}+2 \frac{\left|g^{\prime}\right|^{2}}{\left(1+|g|^{2}\right)^{2}} u=0
$$

A straightforward computation gives that the function

$$
f=\frac{3}{2}\left(\frac{g^{\prime}}{g}\right)^{2}-\frac{g^{\prime \prime}}{g}-\frac{1}{|g|^{2}+1}\left(\frac{g^{\prime}}{g}\right)^{2}
$$

is a bounded complex valued Jacobi function on $\bar{M}$, i.e. its real and imaginary parts satisfy (9). The imaginary part of $f$ is the classical Shiffman's Jacobi function, which expresses the derivative of the planar curvature of horizontal sections with respect to the pure imaginary direction in $\mathbf{C}$ times a positive smooth function; see for instance $[6,22]$. Note that the conormal along the segment $\Gamma$ points to the $x$-axis, where $\xi=x+i y$. A simple calculation shows that

$$
\int_{\Gamma}\left(\frac{\partial f}{\partial \eta} N-f \frac{\partial N}{\partial \eta}\right) d s=\int_{\Gamma}\left(\frac{\partial f}{\partial x} N-f \frac{\partial N}{\partial x}\right) d \xi=0
$$

thus Lemma 4 implies that $\operatorname{Re}(f), \operatorname{Im}(f) \in \mathcal{K}_{0}(M)$. Now our nondegeneracy hypothesis insures that these two functions are linear, so $f=\left\langle N, z_{0}\right\rangle$ for certain $z_{0} \in \mathbf{C}^{3}$. As

$$
\begin{aligned}
\left\langle N, z_{0}\right\rangle & =\frac{2}{1+|g|^{2}}[\operatorname{Re}(a g)+i \operatorname{Re}(b g)]+C \frac{|g|^{2}-1}{|g|^{2}+1} \\
& =\frac{1}{|g|^{2}+1}(A g+B \bar{g})+C \frac{|g|^{2}-1}{|g|^{2}+1},
\end{aligned}
$$

where $a, b, A, B, C$ are complex numbers, we deduce that

$$
\frac{3}{2}\left(\frac{g^{\prime}}{g}\right)^{2}-\frac{g^{\prime \prime}}{g}-\frac{1}{|g|^{2}+1}\left(\frac{g^{\prime}}{g}\right)^{2}=\frac{1}{|g|^{2}+1}(A g+B \bar{g})+C \frac{|g|^{2}-1}{|g|^{2}+1},
$$

and hence

$$
\bar{g}\left(\frac{3}{2} \frac{\left(g^{\prime}\right)^{2}}{g}-g^{\prime \prime}-B-C g\right)=\frac{g^{\prime \prime}}{g}-\frac{1}{2}\left(\frac{g^{\prime}}{g}\right)^{2}+A g-C .
$$

As $g$ is not constant, this equation implies that

$$
\frac{3}{2} \frac{\left(g^{\prime}\right)^{2}}{g}-g^{\prime \prime}-B-C g=0, \quad \frac{g^{\prime \prime}}{g}-\frac{1}{2}\left(\frac{g^{\prime}}{g}\right)^{2}+A g-C=0
$$

thus $\left(g^{\prime}\right)^{2}=g\left(\alpha g^{2}+\beta g+\delta\right)$, with $\alpha, \beta, \delta \in \mathbf{C}$. Hence we have a (possibly branched) holomorphic covering $\pi=\left(g, g^{\prime}\right)$ from $\bar{M}=\mathbf{C} / \mathcal{L}$ onto the Riemann surface $\Sigma=$ $\left\{(z, w) \in \overline{\mathbf{C}}^{2} \mid w^{2}=z\left(\alpha z^{2}+\beta z+\delta\right)\right\}$. We claim the $\Sigma$ has genus one. If $\Sigma$ is a sphere, the total ramification number of $\pi$ is $B(\pi)=2$ degree $(g)$. On the other hand, the pullback by $\pi$ of the meromorphic differential $\frac{d z}{w}$ on $\Sigma$ is $\frac{d g}{g^{\prime}}=d \xi$, which has no poles nor zeroes, so $B(\pi)=\#\left\{\right.$ poles of $\left.\frac{d z}{w}\right\}=2$, which implies that $\pi$ has degree one, a contradiction. Hence $\Sigma$ is a torus and $\pi$ is an unbranched holomorphic covering. Note that the Weierstrass representation [17] $(g, d \xi)$ of $M$ can be induced on $\Sigma$ as $\left(z, c \frac{d z}{w}\right), c \in \mathbf{C}-\{0\}$; thus we obtain a properly embedded minimal torus in certain $\mathbf{R}^{3} / T$ with total curvature $-8 \pi$, which has to be a Riemann example by [14]. This completes the proof. 
Let $\mathcal{G}$ be the 3 -dimensional Lie group of the translations. $\mathcal{G}$ acts in a natural way on $\mathcal{M}$ :

$$
\begin{aligned}
\Psi: \mathcal{G} \times \mathcal{M} & \longrightarrow \mathcal{M}, \\
(\phi, M) & \longmapsto \phi(M) .
\end{aligned}
$$

In order to obtain some regularity behaviour of $\Psi$, we need the corresponding version in our setting of Lemma 8 in [20]:

Lemma 5. Let $\delta>0$ and $M=\widetilde{M} / S \in \mathcal{M}^{*}$, and let $\psi_{t}: \widetilde{M} \longrightarrow \mathbf{R}^{3},|t|<\delta$, be a smooth family of immersions, i.e. $(t, p) \longmapsto \psi_{t}(p)$ is smooth, where $\psi_{t}$ represents an $\mathbf{a}_{t}$-periodic lift of an element $M_{t} \in \mathcal{M}^{*}$ with $M_{0}=M$. Then, the curve $t \longmapsto$ $M_{t} \in \mathcal{M}^{*}$ is smooth and its velocity vector at $t=0$ is $v=\left\langle\left.\frac{d \psi_{t}}{d t}\right|_{t=0}, N\right\rangle \in \mathcal{J}(M)$, $N$ being the Gauss map of $M$.

The proof is analogous to that of Lemma 8 in [20] if we take into account that the surfaces $M_{t}$ for $\delta$ small enough are represented by pairs $\left(\mathbf{a}_{t}, u_{t}\right)$ in an analytic neighbourhood $\mathcal{V}$ of $M$, given by the proof of Theorem 2 , and that $\mathbf{a}_{t}$ depends smoothly on $t$ because, given a point $p \in \widetilde{M}, \mathbf{a}_{t}=\psi_{t}(S p)-\psi_{t}(p)$.

Remark 6. Following the ideas in [20], Remark 2.1, we can deduce that the curve $t \longmapsto M_{t}$ is analytic provided that $(t, p) \longmapsto \psi_{t}(p)$ is also analytic.

As a consequence, the action $\Psi$ is analytic when restricted to $\mathcal{M}^{*}$. Moreover, as the tangent space of $\mathcal{G}$ at the identity $I$ is generated by the infinitesimal translations $\dot{T}_{x}$, along any vector $x$, Lemma 5 gives that $\left(D_{1} \Psi\right)_{(I, M)}\left(\dot{T}_{x}\right)=\langle N, x\rangle$, so $\left(D_{1} \Psi\right)_{(I, M)}$ is injective. This fact implies that the map $\Phi: \mathcal{G} \times \mathcal{M}^{*} \longrightarrow \mathcal{M}^{*} \times \mathcal{M}^{*}$ defined as $(\phi, M) \longmapsto(M, \phi(M))$ is an immersion. We claim that its image is closed in $\mathcal{M}^{*} \times \mathcal{M}^{*}$. For, take sequences $\left\{M_{n}\right\}_{n} \subset \mathcal{M}^{*}$ and $\left\{\phi_{n}\right\}_{n} \subset \mathcal{G}$ such that $M_{n} \longrightarrow$ $M_{\infty} \in \mathcal{M}^{*}$ and $\phi_{n}\left(M_{n}\right) \longrightarrow M_{\infty}^{\prime} \in \mathcal{M}^{*}$. Hence $\phi_{n}$ is a translation of vector $v_{n} \in \mathbf{R}^{3}$. Since for $M \in \mathcal{M}, M \subset \mathbf{R}^{3} / T$, the relation $M+v=M+v+k T$ holds for any $v \in \mathbf{R}^{3}$ and $k \in \mathbf{Z}$, we can suppose without lost of generality that the sequence of the third coordinates $\left\{v_{n, 3}\right\}_{n}$ of $v_{n}$ converges to a real number $v_{\infty, 3}$. As usual, we will use the notation $\widetilde{M}$ for the lift to $\mathbf{R}^{3}$ of the corresponding surface $M \in \mathcal{M}^{*}$. Take a horizontal plane $\Pi$ in $\mathbf{R}^{3}$ such that the intersections $\Pi \cap \widetilde{M}_{\infty}$ and $\left(\Pi+v_{\infty, 3}\right) \cap \widetilde{M}_{\infty}^{\prime}$ are compact. Looking at the sections $\Pi \cap \widetilde{M}_{n}$ and $\left(\Pi+v_{n, 3}\right) \cap\left(\widetilde{M}_{n}+v_{n}\right)$, we deduce that $\left\{v_{n}\right\}_{n}$ is bounded, so our claim holds. By Theorem 16.10.3 in [5], this is equivalent to the existence of a structure of analytic manifold on $\mathcal{M}^{*} / \mathcal{G}$ that makes the projection $\mathcal{M}^{*} \longrightarrow \mathcal{M}^{*} / \mathcal{G}$ a submersion. As the isotropy group at any point of $\mathcal{M}^{*}$ is clearly discrete, this quotient manifold has dimension three.

Note that given $M \in \mathcal{M}$, the flux of its periodic lift $\widetilde{M} \subset \mathbf{R}^{3}$ along a compact horizontal section does not depend on the height of the plane that produces it this is clear for two planes without ends of $\widetilde{M}$ in between; otherwise, as the flux around a planar end vanishes our assertion follows if we compute the flux along the boundary of the possibly nonconnected piece between two sectional horizontal planes and inside a big cylinder parallel to the period vector. This flux vector cannot be horizontal, as can be checked by considering a horizontal transversal section, hence we can define flux $(M)$ as the flux of $\widetilde{M}$ along any horizontal compact section, with positive third coordinate. Now suppose that $M \in \mathcal{M}^{*}, M=\widetilde{M} / S \subset$ $\mathbf{R}^{3} / T$. Let $\Pi$ be a horizontal plane in $\mathbf{R}^{3}$ whose intersection $\Gamma$ with the lifted 
surface is compact, and consider the analytic neighbourhood $\mathcal{V} \subset \mathcal{A} \times \mathcal{U}$ of $M$ in $\mathcal{M}^{*}$ obtained in the proof of Theorem 2, so nearby surfaces in $\mathcal{V}$ are represented by $(\mathbf{a}, u) \equiv M_{\mathbf{a}, u}$. We can suppose that $\Gamma$ is applied on a compact cycle on each $\widetilde{M}_{\mathbf{a}, u}$, and so the flux map can be written as $\operatorname{flux}\left(M_{\mathbf{a}, u}\right)=\int_{\Gamma} \eta_{\mathbf{a}, u} d s_{\mathbf{a}, u}$, with the notation of the proof of Theorem 2. Given an analytic curve $t \longmapsto\left(\mathbf{a}_{t}, u_{t}\right)$ in $\mathcal{V}$, the expression $\eta_{\mathbf{a}_{t}, u_{t}} d s_{\mathbf{a}_{t}, u_{t}}$ depends analytically on $t$; hence the same holds for $\operatorname{flux}\left(M_{\mathbf{a}_{t}, u_{t}}\right)$. As a consequence, the flux map is analytic when restricted to $\mathcal{M}^{*}$. Moreover, Lemma 1 lets us compute its differential at $M$ :

$$
D \operatorname{flux}_{M}(v)=\int_{\Gamma}\left(\frac{\partial v}{\partial \eta} N-v \frac{\partial N}{\partial \eta}\right) d s, \quad \text { for any } v \in \mathcal{J}(M),
$$

$N$ being the Gauss map of $M$. We have another natural analytic map $M \in \mathcal{M}^{*} \mapsto$ $\operatorname{period}(M) \in \mathbf{R}^{3}$, where $\operatorname{period}(M)$ denotes the generator of the cyclic group of translations preserving the lift of $M$, whose third coordinate is positive. The analyticity of this period map follows directly from the proof of Theorem 2 . We can collect these two maps by defining

$$
\begin{aligned}
f: \mathcal{M}^{*} & \longrightarrow \mathbf{R}^{6}, \\
M & \longmapsto
\end{aligned}
$$

Consider on $\mathbf{R}^{6}=\mathbf{R}^{3} \oplus \mathbf{R}^{3}$ the usual symplectic two-form $\Omega$ given by

$$
\Omega\left((\mathbf{a}, \mathbf{b}),\left(\mathbf{a}^{\prime}, \mathbf{b}^{\prime}\right)\right)=\left\langle\mathbf{a}, \mathbf{b}^{\prime}\right\rangle-\left\langle\mathbf{a}^{\prime}, \mathbf{b}\right\rangle,
$$

for any pair of vectors $(\mathbf{a}, \mathbf{b}),\left(\mathbf{a}^{\prime}, \mathbf{b}^{\prime}\right) \in \mathbf{R}^{3} \oplus \mathbf{R}^{3}$. Recall that an immersion of a three-manifold $f: N^{3} \longrightarrow \mathbf{R}^{6}$ is said to be Lagrangian if $f^{*} \Omega=0$.

If $(\dot{\mathbf{a}}, \dot{u}) \in \operatorname{Kernel}\left(D f_{M}\right)$, then $\dot{\mathbf{a}}=0$ and $\dot{u} \in \mathcal{K}(M)$, so (11) implies that $\int_{\Gamma}\left(\frac{\partial \dot{u}}{\partial \eta} N-\dot{u} \frac{\partial N}{\partial \eta}\right) d s=0$; hence from Lemma 4 we have $\operatorname{Kernel}\left(D f_{M}\right)=\mathcal{K}_{0}(M)$. On the other hand, Lemma 3 yields $f^{*} \Omega=0$. It is also clear that $f$ can be induced on the quotient manifold $\mathcal{M}^{*} / \mathcal{G}$. These facts are summarized in the following result:

Theorem 4. Suppose that $\mathcal{M}^{*}$ is nonvoid. Then, $\mathcal{M}^{*} / \mathcal{G}$ is a real analytic threedimensional manifold, and the map $f: \mathcal{M}^{*} / \mathcal{G} \longrightarrow \mathbf{R}^{6}$ given by

$$
f([M])=(\operatorname{period}(M), \operatorname{flux}(M)),
$$

$[M]$ being the class of $M$ in $\mathcal{M}^{*} / \mathcal{G}$, is an analytic Lagrangian immersion. Also, the tangent space $T_{[M]}\left(\mathcal{M}^{*} / \mathcal{G}\right)$ can be naturally identified with $\mathcal{J}(M) / \mathcal{K}_{0}(M)$.

Remark 7 . The period map is a local parameter for $\mathcal{M}^{*} / \mathcal{G}$ around an element $[M]$ if and only if the only well-defined bounded Jacobi functions on $M$ come from translations in $\mathbf{R}^{3}$. This situation occurs for instance in the Riemann examples.

\section{THE SCREW MOTION CASE}

We can study in a similar way the space of proper minimal embeddings which are invariant by a nontrivial screw motion. Let $S_{\theta, \lambda}$ be the screw motion obtained by composition of a rotation $R_{\theta}$ of angle $\left.\theta \in\right] 0,2 \pi\left[\right.$ around the $x_{3}$-axis followed by a translation of vector $\lambda e_{3}, \lambda \in \mathbf{R}-\{0\}$. Let $\psi: \widetilde{M} \longrightarrow \mathbf{R}^{3}$ be a proper minimal embedding such that there exists a conformal diffeomorphism $S$ of $\widetilde{M}$ with $\psi \circ S=$ $S_{\theta, \lambda} \circ \psi$, so $\psi$ induces a properly embedded minimal surface $M=\widetilde{M} / S \subset \mathbf{R}^{3} / S_{\theta, \lambda}$. Assume that $M$ is orientable and has finite topology, $M=\bar{M}-\left\{p_{1}, \ldots, p_{r}\right\}$. We no longer have a well-defined Gauss map on $M$, but the Gauss map $N$ of $\psi$ satisfies $N \circ S=R_{\theta} \circ N$. However, thanks to the work of Meeks and Rosenberg [15] we 
know that $M$ has finite total curvature and a controlled behaviour at infinity: All its ends are simultaneously asymptotic to horizontal planes, flat vertical annuli or ends of helicoids. Suppose that we are in the first case. As the ideas we are going to express are similar to the ones in the $\mathbf{R}^{3} / T$-case, we will give a brief outline of the theory, emphasizing only the new details that appear in this case.

Fix $\psi: \widetilde{M} \longrightarrow \mathbf{R}^{3}, M=\widetilde{M} / S \subset \mathbf{R}^{3} / S_{\theta, \lambda}$ as before. First of all, there exist a neighbourhood $\mathcal{A}$ of $(\theta, \lambda)$ in $\mathbf{R}^{2}$ and a $C^{\infty}$-family of singly-periodic proper immersions $\psi_{\theta^{\prime}, \lambda^{\prime}}: \widetilde{M} \longrightarrow \mathbf{R}^{3},\left(\theta^{\prime}, \lambda^{\prime}\right) \in \mathcal{A}$, such that $\psi_{\theta, \lambda}=\psi, \psi_{\theta^{\prime}, \lambda^{\prime}} \circ S=S_{\theta^{\prime}, \lambda^{\prime}} \circ \psi_{\theta^{\prime}, \lambda^{\prime}}$, $\psi_{\theta^{\prime}, \lambda^{\prime}}$ is minimal outside a solid cylinder and its ends are essentially the same as those of $\psi$. Its Gauss map $N_{\theta^{\prime}, \lambda^{\prime}}$ satisfies $N_{\theta^{\prime}, \lambda^{\prime}} \circ S=R_{\theta^{\prime}} \circ N_{\theta^{\prime}, \lambda^{\prime}}$, and as each end of $\psi_{\theta^{\prime}, \lambda^{\prime}}$ is planar we can modify $N_{\theta^{\prime}, \lambda^{\prime}}$, defining a new transverse map $\widetilde{N}_{\theta^{\prime}, \lambda^{\prime}}$ on $\widetilde{M}$ such that $\widetilde{N}_{\theta^{\prime}, \lambda^{\prime}} \circ S=R_{\theta^{\prime}} \circ \widetilde{N}_{\theta^{\prime}, \lambda^{\prime}}, \quad \widetilde{N}_{\theta^{\prime}, \lambda^{\prime}}=\frac{1}{\left\langle N, e_{3}\right\rangle} e_{3}$ around each end-note that this last equation is compatible with the behaviour of $N$ and of $\widetilde{N}_{\theta^{\prime}, \lambda^{\prime}}$ with respect to $S$-and $\langle\widetilde{N}, N\rangle \equiv 1$ on $\widetilde{M}$, where $\widetilde{N}=\widetilde{N}_{\theta, \lambda}$ and $N$ is the Gauss map of $\psi$. Given $u \in C^{2, \alpha}(\bar{M})$ small enough, $\psi_{\theta^{\prime}, \lambda^{\prime}}+u \widetilde{N}_{\theta^{\prime}, \lambda^{\prime}}$ is a proper immersion such that $\left(\psi_{\theta^{\prime}, \lambda^{\prime}}+u \widetilde{N}_{\theta^{\prime}, \lambda^{\prime}}\right) \circ S=S_{\theta^{\prime}, \lambda^{\prime}} \circ\left(\psi_{\theta^{\prime}, \lambda^{\prime}}+u \widetilde{N}_{\theta^{\prime}, \lambda^{\prime}}\right)$, so it induces a properly immersed surface in $\mathbf{R}^{3} / S_{\theta^{\prime}, \lambda^{\prime}}$. The mean curvature $H\left(\psi_{\theta^{\prime}, \lambda^{\prime}}+u \widetilde{N}_{\theta^{\prime}, \lambda^{\prime}}\right)$ is well-defined on $M$; thus we can consider the operator $\bar{H}\left(\theta^{\prime}, \lambda^{\prime}, u\right)=\lambda H\left(\psi_{\theta^{\prime}, \lambda^{\prime}}+u \widetilde{N}_{\theta^{\prime}, \lambda^{\prime}}\right)$ (where $\lambda$ is a conformal factor as in the translation case), which is a $C^{\alpha}(\bar{M})$-valued analytic operator on the product $\mathcal{A} \times \mathcal{U}$ of certain neighbourhoods of $(\theta, \lambda)$ in $\mathbf{R}^{2}$ and of zero in $C^{2, \alpha}(\bar{M})$. If we take a curve $(\theta(t), \lambda(t), u(t))$ in $\mathcal{A} \times \mathcal{U}$ such that $(\theta(0), \lambda(0), u(0))=(\theta, \lambda, 0)$, the variational field $V$ of $\psi_{\theta(t), \lambda(t)}+u(t) \widetilde{N}_{\theta(t), \lambda(t)}$ satisfies $\langle V, N\rangle=\langle\dot{\psi}, N\rangle+\dot{u}$, where the dot means derivative respect to $t$ at $t=0$ and $\dot{\psi}=\left.\frac{d}{d t}\right|_{t=0} \psi_{\theta(t), \lambda(t)} . \dot{u}$ is well-defined on $\bar{M}$, but the other term in $\langle V, N\rangle$ has the following multivaluation:

$$
\langle\dot{\psi}, N\rangle \circ S=\langle\dot{\psi}, N\rangle+\dot{\theta} \operatorname{det}\left(p, N, e_{3}\right)+\dot{\lambda}\left\langle e_{3}, N\right\rangle
$$

$p$ being the position vector on $\widetilde{M}$. As $L\left(\operatorname{det}\left(p, N, e_{3}\right)\right)=L\left\langle e_{3}, N\right\rangle=0, L$ being the Jacobi operator of $\psi$, the function $L(\langle\dot{\psi}, N\rangle+\dot{u})$ is well-defined on $M$ and $D \bar{H}_{(\theta, \lambda, 0)}(\dot{\theta}, \dot{\lambda}, \dot{u})=\frac{1}{2} \lambda L(\langle\dot{\psi}, N\rangle+\dot{u})$, for any $(\dot{\theta}, \dot{\lambda}, \dot{u}) \in \mathbf{R}^{2} \times C^{2, \alpha}(\bar{M})$. Put $V=$ $V(M)=\left\{\langle\dot{\psi}, N\rangle=\left\langle\left.\frac{d}{d t}\right|_{t=0} \psi_{\theta(t), \lambda(t)}, N\right\rangle /(\dot{\theta}, \dot{\lambda}) \in \mathbf{R}^{2}\right\} \subset C^{\infty}(\widetilde{M})$ - which can be naturally identified with $\mathbf{R}^{2}$ - and define $\mathcal{B}=\mathcal{B}(M)=V \oplus C^{2, \alpha}(\bar{M}) \subset C^{2, \alpha}(\widetilde{M})$ and the compactified Jacobi operator $\bar{L}=\lambda L=2 D \bar{H}_{(\theta, \lambda, 0)}: \mathcal{B}(M) \longrightarrow C^{\alpha}(\bar{M})$.

As in the previous section, we can consider the flux of a surface along an oriented horizontal compact section, which does not depend on the height. At this point we should consider the torque map along such section, defined as

$$
\operatorname{torque}\left(\psi_{t}\right)=\int_{\Gamma} \psi_{t} \wedge \eta_{t} d s_{t},
$$

for a deformation $\psi_{t}$ of our original periodic minimal surface $\psi$ by immersions with ends asymptotic to horizontal planes. In fact, we are interested in the third coordinate of this torque vector for a minimal surface, which does not depend on the height of the compact section $\Gamma$ - for two such sections with no ends in between, this assertion holds because the $\mathbf{R}^{3}$-valued differential $\psi \wedge D \psi$ has divergence zero; the horizontality of the torque vector at a planar type end with vertical limit normal vector, see for instance [12] or [18], implies the property in the general case. Arguments similar to the ones in Lemma 1 let us compute the derivative of the 
torque map, obtaining

$$
\begin{aligned}
\left.\frac{d}{d t}\right|_{t=0} \operatorname{torque}\left(\psi_{t}\right)= & -\int_{\Gamma}\langle V, N\rangle D \psi\left(\gamma^{\prime}\right) d s \\
& +\int_{\Gamma} \psi \wedge\left(\frac{\partial}{\partial \eta}(\langle V, N\rangle) N-\langle V, N\rangle \frac{\partial N}{\partial \eta}\right) d s,
\end{aligned}
$$

where, as usual, $V$ is the variational field of $\psi_{t}$ and $\gamma(s)$ is a parametrization by the $\psi$-arc length of $\Gamma$. Since the first integral is horizontal, it is easy to deduce the following expression for the derivative of the third coordinate of the torque map:

$$
\begin{aligned}
& \left.\frac{d}{d t}\right|_{t=0} \operatorname{torque}_{3}\left(\psi_{t}\right) \\
& \quad=\int_{\Gamma}\left(\frac{\partial}{\partial \eta}(\langle V, N\rangle) \operatorname{det}\left(p, N, e_{3}\right)-\langle V, N\rangle \frac{\partial}{\partial \eta}\left(\operatorname{det}\left(p, N, e_{3}\right)\right)\right) d s
\end{aligned}
$$

If we define $\mathcal{J}(M), \mathcal{K}(M)$ and $\mathcal{K}_{0}(M)$ as in the translation case - note that the space $\mathcal{B}(M)$ has changed-we can prove analogously that

$$
\mathcal{K}_{0}(M)=\left\{\begin{array}{l|l}
u \in \mathcal{K}(M) & \begin{array}{l}
\left.\int_{\Gamma} \frac{\partial u}{\partial \eta} \operatorname{det}-u \frac{\partial \mathrm{det}}{\partial \eta}\right) d s=0, \\
\int_{\Gamma}\left(\frac{\partial u}{\partial \eta}\left\langle N, e_{3}\right\rangle-u \frac{\partial\left\langle N, e_{3}\right\rangle}{\partial \eta}\right) d s=0
\end{array}
\end{array}\right\},
$$

where det denotes the function $\operatorname{det}\left(p, N, e_{3}\right)$, and that $\operatorname{dim} \mathcal{J}(M)=2+\operatorname{dim} \mathcal{K}_{0}(M)$. From this description of $\mathcal{K}_{0}(M)$, Lemma 1 and (13) one has $\operatorname{det}\left(p, N, e_{3}\right),\left\langle N, e_{3}\right\rangle \in$ $\mathcal{K}_{0}(M)$; hence the space of Jacobi functions $\mathcal{J}(M)$ has at least dimension four. Our minimal surface $M$ will be called nondegenerate when $\mathcal{K}_{0}(M)$ has dimension two.

In the space $\mathcal{M}$ of connected nonflat properly embedded minimal surfaces in quotients of $\mathbf{R}^{3}$ by nontrivial screw motions $S_{\theta, \lambda}$ - depending on the surface-, with fixed finite topology and planar ends we can define two natural senses of convergence: take surfaces $M_{n} \subset \mathbf{R}^{3} / S_{\theta_{n}, \lambda_{n}}, M_{\infty} \subset \mathbf{R}^{3} / S_{\theta_{\infty}, \lambda_{\infty}}$ in $\mathcal{M}, n=1,2, \ldots$ The notion of smooth convergence of $\left\{M_{n}\right\}_{n}$ to $M_{\infty}$ coincides with the one expounded in section 3. Denoting by $\bar{M}_{n}, \bar{M}_{\infty}$ the compactifications of $M_{n}=\widetilde{M}_{n} / S_{\theta_{n}, \lambda_{n}}$ and $M_{\infty}=\widetilde{M}_{\infty} / S_{\theta_{\infty}, \lambda_{\infty}}$, and using the $C^{\infty}$-family $\psi_{\theta^{\prime}, \lambda^{\prime}}$ at the beginning of this section around $M_{\infty}$, we will say that the sequence $\left\{\bar{M}_{n}\right\}_{n}$ converges to $\bar{M}_{\infty}$ smoothly if $\left(\theta_{n}, \lambda_{n}\right) \rightarrow\left(\theta_{\infty}, \lambda_{\infty}\right)$, there exists a sequence $\left\{\widetilde{N}_{n}\right\}_{n} \subset C^{\infty}\left(\widetilde{M}, \mathbf{R}^{3}\right)$ such that $\widetilde{N}_{n}=\frac{1}{\left\langle N_{\infty}, e_{3}\right\rangle} e_{3}$ in a neighbourhood of each end, $\widetilde{N}_{n} \circ S=R_{\theta_{n}} \circ \widetilde{N}_{n}$ in $\widetilde{M}$ and there exists a sequence $\left\{u_{n}\right\}_{n} \subset C^{\infty}\left(\bar{M}_{\infty}\right)$ such that $\widetilde{M}_{n}$ can be expressed as $\psi_{\theta_{n}, \lambda_{n}}+u_{n} \widetilde{N}_{n}$ with $u_{n} \rightarrow 0$ in $C^{k}\left(\bar{M}_{\infty}\right)$, for any $k \geq 0$. Theorem 1 extends to this situation, insuring that these two notions of convergence are equivalent for sequences in $\mathcal{M}$ with $\left(\theta_{n}, \lambda_{n}\right)$ converging to $\left(\theta_{\infty}, \lambda_{\infty}\right)$; hence the second one is independent of the families $\psi_{\theta^{\prime}, \lambda^{\prime}}, \widetilde{N}_{n}$. We will also denote by $\mathcal{M}^{*}$ the set of nondegenerate surfaces invariant by nontrivial screw motions $\left.S_{\theta, \lambda},(\theta, \lambda) \in\right] 0,2 \pi[\times(\mathbf{R}-\{0\})$. The structure result in this situation is

Theorem 5. $\mathcal{M}^{*}$ is open in $\mathcal{M}$. In the nonvoid case, $\mathcal{M}^{*}$ is a four dimensional real analytic manifold.

The proof of this result is similar to the one in the translation case. The only differences occur when we need to prove the orthogonality of the mean curvature 
and the function $\operatorname{det}\left(p, N, e_{3}\right)$, and the compatibility of the locally defined analytic structures. The first problem can be solved by reasoning as in [20], Lemma 7, and for the second one we use the corresponding analytic immersion $j$ defined on a neighbourhood $\mathcal{V}$ of an element in $\mathcal{M}^{*}$ that takes nondegenerate minimal surfaces $M_{\theta^{\prime}, \lambda^{\prime}, u}$ in this neighbourhood into $\left(\theta^{\prime}, \lambda^{\prime}, u\right) \in \mathbf{R}^{2} \times C^{2, \alpha}(\{|w|<\varepsilon\})$.

Remark 8. In the case $\theta=0$, we again find surfaces $M \subset \mathbf{R}^{3} / T, T$ being a vertical translation with finite topology and planar ends. For such a surface, its Gauss map is well defined, and it is easy to prove that $\left\langle N, e_{1}\right\rangle,\left\langle N, e_{2}\right\rangle,\left\langle N, e_{3}\right\rangle, \operatorname{det}\left(p, N, e_{3}\right)$ belong to $\mathcal{K}_{0}(M)$ if flux $(M)$ is vertical, and $\left\langle N, e_{3}\right\rangle,\langle N, \operatorname{flux}(M)\rangle, \operatorname{det}\left(p, N, e_{3}\right) \in$ $\mathcal{K}_{0}(M)$ otherwise, where flux $(M)$ is defined as in section 4 . This implies that the space of nondegenerate minimal surfaces invariant by screw motions with planar ends cannot be a manifold around an example with $\theta=0$.

Lemma 5 (resp. Remark 6) can be also extended to the screw motion case, expressing the smoothness (resp. analyticity) in the manifold $\mathcal{M}^{*}$ in terms of the lifted surfaces in $\mathbf{R}^{3}$. The only novelty in its proof is that surfaces $M_{t}$ near $M \subset \mathbf{R}^{3} / S_{\theta, \lambda}$ in $\mathcal{M}^{*}$ can be represented by elements $\left(\theta_{t}^{\prime}, \lambda_{t}^{\prime}, u_{t}\right)$ in an analytic neighbourhood of $(\theta, \lambda, 0)$, where $\theta_{t}^{\prime}, \lambda_{t}^{\prime}$ depend smoothly (resp. analytically) on $t$ because $\lambda_{t}^{\prime}=\left\langle\psi_{t}(S p)-\psi_{t}(p), e_{3}\right\rangle$ and $\left(x_{t}^{2}(p)+y_{t}^{2}(p)\right) \sin \theta_{t}^{\prime}=-\operatorname{det}\left(\psi_{t}(S p), \psi_{t}(p), e_{3}\right)$ for a generic point $p$ in $\widetilde{M}$ (here $x_{t}, y_{t}$ are the first two components of the lift $\psi_{t}$ of $M_{t}$ and we continue using the same notation as before). Moreover, denoting by $\mathcal{G}$ the 2 -dimensional group of the vertical screw motions, the analytic structure on $\mathcal{M}^{*} / \mathcal{G}$ follows as in the translation case, but now it has dimension two. This quotient manifold can be also immersed in a Lagrangian way into $\mathbf{C}^{2}=\mathbf{R}^{2} \oplus \mathbf{R}^{2}$ by means of the map $f: \mathcal{M}^{*} / \mathcal{G} \longrightarrow \mathbf{R}^{4}$ given by $f([M])=\left(\operatorname{screw}(M), \operatorname{torque}_{3}(M), \operatorname{flux}_{3}(M)\right)$, where $\operatorname{screw}(M)=(\theta, \lambda)$ if $M \subset \mathbf{R}^{3} / S_{\theta, \lambda}, \lambda>0$, and the subindex $\bullet_{3}$ denotes the third coordinate of the corresponding vector.

\section{REFERENCES}

1. R. Bohme \& A. J. Tromba, The index theorem for classical minimal surfaces, Ann. of Math. 113 (1981) 447-499. MR 83a:58031

2. M. Callahan, D. Hoffman \& H. Karcher, A family of singly-periodic minimal surfaces invariant under a screw-motion, Experiment. Math. 2 (1993) 157-182. MR 95h:53007

3. M. Callahan, D. Hoffman \& W. H. Meeks III, Embedded minimal surfaces with an infinite number of ends, Invent. Math. 96 (1989) 459-505. MR 90b:53005

4. M. Callahan, D. Hoffman \& W. H. Meeks III, The structure of singly periodic minimal surfaces, Invent. Math. 99 (1990) 455-481. MR 92a:53005

5. J. Dieudonné, Éléments d'Analyse, vol. III Gauthier-Villars, Paris (1970). MR 42:5266

6. Y. Fang \& F. Wei, On uniqueness of Riemann's examples, Preprint.

7. D. Gilbarg \& N. S. Trudinger, Elliptic Partial Differential Equations of Second Order, 2nd ed., Springer-Verlag (1983). MR 86c:35035

8. D. Hoffman \& H. Karcher, Complete embedded minimal surfaces of finite total curvature, preprint.

9. D. Hoffman \& W. H. Meeks III, Minimal surfaces based on the catenoid, Amer. Math. Monthly 97 (1990) 702-730. MR 92c:53002

10. D. Hoffman \& W. H. Meeks III, The strong halfspace theorem for minimal surfaces, Invent. Math. 101 (1990) 373-377. MR 92e:53010

11. H. Karcher, Embedded minimal surfaces derived from Scherk's examples, Manuscripta Math. 62 (1988) 83-114. MR 89i:53009

12. R. Kusner, Global geometry of extremal surfaces in three-space, PhD Thesis, University of California, Berkeley (1988).

13. R. Langevin \& H. Rosenberg, A maximum principle at infinity for minimal surfaces and applications, Duke Math. J. 57 (1988) 819-828. MR 90c:53025 
14. F. J. López, M. Ritoré \& F. Wei, A characterization of Riemann's minimal surfaces, to appear in J. Differential Geometry.

15. W. H. Meeks III \& H. Rosenberg, The geometry of periodic minimal surfaces, Comm. Math. Helv. 68 (1993) 538-578. MR 95a:53011

16. S. Montiel \& A. Ros, Schrödinger operators associated to a holomorphic map, Proceedings Conference on Global Differential Geometry and Global Analysis, Berlin, 1990, Lecture Notes in Math. 1481 147-174. MR 93k:58053

17. R. Osserman, A survey of minimal surfaces, vol. 1, Cambridge Univ. Press, New York (1989).

18. J. Pérez. Riemann bilinear relations on minimal surfaces, preprint.

19. J. Pérez \& A. Ros, Some uniqueness and nonexistence theorems for embedded minimal surfaces, Math. Ann. 295 (1993) 513-525. MR 94a:53020

20. J. Pérez \& A. Ros, The space of properly embedded minimal surfaces with finite total curvature, Indiana Univ. Math. J. 45 (1996) 177-204. CMP 1996:17

21. B. Riemann, Gesammelte mathematische Werke, 2nd edition, B.G. Teubner, Leipzig (1892).

22. M. Shiffman, On surfaces of stationary area bounded by two circles, or convex curves, in parallel planes, Ann. of Math. 63 (1956) 77-90. MR 17:632d

23. F. Tomi \& A. J. Tromba, Extreme curves bound embedded minimal surfaces of the type of the disc, Math. Z. 158 (1978) 137-145. MR 80k:53011

24. A. J. Tromba, Degree theory on oriented infinite dimensional varieties and the Morse number of minimal surfaces spanning a curve in $\mathbf{R}^{n}$. Part I: $n \geq 4$, Trans. Amer. Math. Soc. 290 (1985) 385-413. MR 87d:58041

25. B. White, The space of $m$-dimensional surfaces that are stationary for a parametric elliptic functional, Indiana Univ. Math. J. 36 (1987) 567-603. MR 88k:58027

26. B. White, The space of minimal submanifolds for varying Riemannian metrics, Indiana Univ. Math. J. 40 (1991) 161-200. MR 92i:58028

Departamento de Geometría y Topología, Facultad de Ciencias, Universidad de Granada, 18071 Granada, SPAIN

E-mail address: jperez@goliat.ugr.es 